\title{
New Metrics for Multiple Testing with Correlated Outcomes
}

\author{
Maya B. Mathur ${ }^{*}$ \& Tyler J. VanderWeele ${ }^{2}$ \\ ${ }^{1}$ Quantitative Sciences Unit and Department of Pediatrics, Stanford University, Palo Alto, CA, USA \\ ${ }^{2}$ Department of Epidemiology, Harvard T. H. Chan School of Public Health, Boston, MA, USA
}

\begin{abstract}
We propose new metrics comparing the observed number of hypothesis test rejections $(\hat{\theta})$ at an unpenalized $\alpha$-level to the distribution of rejections that would be expected if all tested null hypotheses held (the "global null"). Specifically, we propose reporting a "null interval" for the number of $\alpha$-level rejections expected to occur in $95 \%$ of samples under the global null, the difference between $\hat{\theta}$ and the upper limit of the null interval (the "excess hits"), and a one-sided joint test based on $\hat{\theta}$ of the global null. For estimation, we describe resampling algorithms that asymptotically recover the sampling distribution under the global null. These methods accommodate arbitrarily correlated test statistics and do not require high-dimensional analyses. In a simulation study, we assess properties of the proposed metrics under varying correlation structures as well as their power for outcome-wide inference relative to existing methods for controlling familywise error rate. We provide an R package, NRejections. Ultimately, existing procedures for multiple hypothesis testing typically penalize inference in each test, which is useful to temper interpretation of individual findings; yet on their own, these procedures do not fully characterize global evidence strength across the multiple tests. Our new metrics help remedy this limitation.
\end{abstract}

Keywords: multiplicity, Type I error, bootstrap, resampling, familywise error rate 


\section{INTRODUCTION}

In studies testing multiple hypotheses, the problem of inflated Type I error rates is usually handled, if at all, through procedures that preserve familywise error rate (FWER) or false discovery rate $(\mathrm{FDR})$ by penalizing individual $p$-values or critical values. These procedures can be valuable for individually correcting inference for each hypothesis test. However, as standalone reporting methods, they may provide incomplete insight into the overall strength of evidence across tests. For example, if individual hypothesis tests of the associations between a single exposure of interest and 40 outcome measures result in a total of 10 rejections at an uncorrected $\alpha=0.05$ and result in 1 rejection at a Bonferroni-corrected $\alpha \approx 0.001$, how strong is the overall evidence supporting associations between the exposure and the outcomes, considered jointly? Given only the information typically reported in corrected or uncorrected multiple tests, such questions can be hard to answer.

Intuitive speculation about overall evidence strength becomes especially challenging when the hypothesis tests are correlated, which is typically the case when related research questions are considered (Blakesley et al. 2009) or in "outcome-wide" analyses that assess associations between a single exposure and a number of outcomes (VanderWeele, 2017; VanderWeele et al. 2020). Indeed, as we will illustrate, the results of a given set of individual tests (whether multiplicity-corrected or not) may be strongly suggestive of at least some genuine effects if the tests are independent, but may be entirely consistent with chance (in a manner we will formalize) if the tests are correlated. In practice, the correlation structure of the tests is usually unknown, further impeding intuitive assessment.

We therefore aim to supplement existing multiple-testing procedures (e.g., Dunn (1961); Holm (1979); Romano \& Wolf (2005a, 2007); Westfall \& Young (1993)) with simple metrics that directly characterize overall evidence strength while accommodating arbitrarily correlated test statistics. These metrics focus on the total number of hypothesis test rejections at an arbitrary $\alpha$ level (such as the usual, uncorrected $\alpha=0.05$ ). First, we propose reporting a null interval representing a plausible range for the total number of rejections in $95 \%$ of samples that would occur if all null hypotheses were true (a scenario we call the "global null"), along with the difference between the number of observed rejections and the upper interval limit (the excess hits). For example, if we reject 10 of 40 hypotheses at $\alpha=0.05$, we might be tempted to conclude intuitively that this is "more" than the expected $0.05 \times 40=2$ rejections. However, if the null interval is $[0,11]$, accounting for correlation between the tests, this would suggest little evidence overall for genuine associations across the 40 tests. In contrast, if we instead reject 18 tests under the same correlation structure, the null interval indicates that we have observed 7 excess hits beyond the number that would be expected in $95 \%$ of samples generated under the global null, which is suggestive of strong overall evidence that at least some of the tested associations are present. Additionally, we propose using the number of rejections to conduct a one-sided global test of the global null, whose $p$-value represents the probability, in samples generated under the global null, of observing at least as many $\alpha$-level rejections as were actually observed. 
Although standard methods for FWER control are not explicitly designed to characterize overall evidence strength, they could in principle be repurposed into a global test. That is, letting $\alpha_{W}$ denote the level of familywise inference, rejection of at least one test with inference corrected to preserve a familywise $\alpha_{W}=0.05$ implies rejection of the global null at $\alpha_{W}=0.05$. Several existing methods strongly control FWER in hypothesis tests with unknown correlation structure and could therefore be suitable for a global test. The most widespread approaches are the classical one-step Bonferroni correction (Dunn, 1961) and its uniformly more powerful successor, the step-down Holm method (Holm, 1979), both of which can be computed in closed form. By avoiding specifying or estimating the correlation structure, these naïve methods accommodate even worst-case correlation structures but can yield conservative inference. Other closed-form methods achieve better power by assuming independence (e.g., various procedures based on Simes (1986)'s inequality) or known logical dependencies between tests (e.g., Shaffer (1986)) but can produce anticonservative inference if these assumptions are violated (Shaffer, 1995). We focus here on modern methods, detailed in Section 2, that avoid such assumptions by empirically estimating the correlation structure via resampling (Romano \& Wolf, 2005a, 2007; Westfall \& Young, 1993). Related methods control FDR rather than FWER (e.g., Romano \& Wolf (2007); van der Laan et al. (2005, 2004)), but because FDR control does not appear to have a direct relationship with the null interval and excess hits metrics discussed in the present paper, we do not further consider these methods. Alternative approaches are designed for a large number of hypothesis tests, as in high-dimensional genetic studies (e.g., Friguet et al. (2009); Leek \& Storey (2008); Sun \& Cai (2009)); however, because correlated hypothesis tests can be particularly problematic in traditional low-dimensional settings (Clarke et al., 2009), we aim to provide methods that apply regardless of the number of tests.

In this paper, we first derive assumptions for the asymptotic validity of a resampling-based null interval, the corresponding excess hits, and a global test of the number of rejections, and we describe specific resampling algorithms fulfilling these assumptions. Second, we conduct a simulation study in which we (1) compare the null interval to the observed number of rejections for varying effect sizes; and (2) assess the relative power of global tests and the number of rejected null hypotheses at a familywise-controlled $\alpha_{W}=0.05$ using the number of rejections or derived from existing FWER-control methods, as discussed above. To our knowledge, prior simulation studies of existing FWER methods have not reported on their performance as global tests (Romano \& Wolf, 2007). We illustrate application of our proposed metrics through an applied example.

\section{EXISTING RESAMPLING-BASED MULTIPLICITY CORRECTIONS}

Table 1 summarizes existing methods that strongly control FWER for arbitrarily correlated tests. Westfall \& Young (1993) propose resampling algorithms that resemble the standard bootstrap, but that modify the data in order to enforce the global null (an approach 
similar to what we will adopt). For example, suppose we conduct one-sample $t$-tests on the potentially correlated variables $\left(Y_{1}, \cdots, Y_{W}\right)$ with the global null stating that $E\left[Y_{w}\right]=$ $0 \forall w \in\{1, \cdots, W\}$. To resample under the global null, the observed data could first be centered by their respective sample means, then resampled with replacement (Westfall \& Young, 1993). Thus, in the resampled datasets, the global null holds regardless of the true parameters $\left(E\left[Y_{1}\right], \cdots, E\left[Y_{W}\right]\right)$ underlying the original sample. Then, Westfall \& Young (1993)'s one-step "minP" method and uniformly more powerful step-down variant (here termed "Wstep") adjust the observed $p$-values using quantiles of the distribution of $p$-values calculated in the resamples.

Other FWER methods use parametric resampling approaches that do not enforce the global null in the resampled data, but rather that generate datasets resembling the original data (Romano \& Wolf, 2005a, 2007). Essentially invoking the duality of hypothesis tests and confidence intervals, the resampled test statistics are then centered by their estimated values in the observed data in order to recover the null distribution; other related methods showed less favorable performance in prior simulations, so we do not further consider them here (Romano \& Wolf, 2007, van der Laan et al., 2004).

This latter class of resampling approaches obviates a key assumption used to simplify computation in the minP and Wstep approaches. This "subset pivotality" assumption has been discussed at length elsewhere (e.g., Romano \& Wolf (2005a); Westfall \& Troendle (2008); Westfall \& Young (1993); Yekutieli \& Benjamini (1999)); it states that for any subset $K$ of hypotheses being tested, if all null hypotheses in $K$ hold, then the joint distribution of test statistics in $K$ is the same regardless of the truth or falsehood of all hypotheses not in $K$ (Westfall \& Troendle, 2008). (See Definition 5.1 below for a more formal definition.) The motivation for subset pivotality is that strong FWER-control methods that empirically estimate the correlation structure must control FWER not only when the global null holds, but also for any configuration of true and false null hypotheses. Although it might appear that resamples would therefore need to be generated under every such configuration, Westfall \& Young (1993)'s methods circumvent this problem, requiring only one set of resamples under the global null, by invoking subset pivotality.

Subset pivotality can fail, for example, when testing pairwise correlations of three variables, $X, Y$, and $Z$ (Romano \& Wolf (2005a)'s Example 4.1). In this setting, the joint distribution of the statistics $\hat{\rho}_{X Y}$ and $\hat{\rho}_{X Z}$ when a particular subset $K$ of the null hypotheses hold, namely $\rho_{X Y}=\rho_{X Z}=0$, depends on $\rho_{Y Z}$ and hence on the truth or falsehood of a hypothesis not in $K$ (Romano \& Wolf, 2005a). Thus, under Westfall \& Young (1993)'s resampling approach, $\hat{\rho}_{X Y}^{(j)}$ and $\hat{\rho}_{X Z}^{(j)}$ would be correctly centered at 0 , but they would be independent because the global null is enforced. In contrast, under Romano \& Wolf (2007)'s resampling approach, $\hat{\rho}_{X Y}^{(j)}$ and $\hat{\rho}_{X Z}^{(j)}$ would likewise be centered at 0 because they would have been centered by the sample estimates $\hat{\rho}_{X Y}$ and $\hat{\rho}_{X Z}$, but they would also be correlated to an extent determined by $\rho_{Y Z}$. In turn, the distribution of the maximum test statistic depends on the joint distribution of $\left(\hat{\rho}_{X Y}, \hat{\rho}_{X Z}\right)$. Importantly, subset pivotality will not be required for our proposed methods: unlike FWER-control methods, our proposed methods concern only the global null, and thus 
even when subset pivotality does not hold, it is sufficient to estimate via resampling the single sampling distribution of the test statistics under the global null. To build upon these existing methods by directly characterizing global evidence strength, we now develop theory underlying our proposed metrics.

Table 1: Selected existing methods for strong FWER control with correlated hypothesis tests

\begin{tabular}{lll}
\hline Method & Type & Means of handling correlation \\
\hline Bonferroni & 1-step & Conservatively making no assumptions \\
Holm & Step-down & Conservatively making no assumptions \\
minP & 1-step & $\begin{array}{l}\text { Resampling under global null to } \\
\text { estimate correlation structure }\end{array}$ \\
Wstep & Step-down & Same as above \\
Romano & Step-down & $\begin{array}{l}\text { Resampling without restrictions to } \\
\text { estimate correlation structure }\end{array}$ \\
\hline
\end{tabular}

\section{SetTing AND NOTATION}

Suppose that $K$ random variables are measured on $N$ subjects, with the resulting matrix denoted $Z \in \mathbb{R}^{N \times K}$. Let $Z_{n k}$ denote, for the $n^{\text {th }}$ subject, the $k^{\text {th }}$ random variable. Consider a resampling algorithm that generates, for iterate $j$, a dataset $Z^{(j)} \in \mathbb{R}^{N \times K}$ containing the random vector $\left(Z_{n 1}^{(j)}, \cdots, Z_{n K}^{(j)}\right)$ for each subject $n$. There are a total of $B$ resampled datasets. We use the superscript "(j)" to denote random variables, distributions, and statistics in resampled dataset $j$. Further suppose that we conduct $W$ tests of point null hypotheses, each at level $\alpha$. Denote the $w^{\text {th }}$ null hypothesis as $H_{0 w}$. Let $c_{w, \alpha}$ be the critical value for the test statistic, $T_{w}$, of the $w^{t h}$ test. The $W$-vector of test statistics is $T=\left(T_{1}, \cdots, T_{w}\right)$. We define the "global null" as the case in which all $W$ null hypotheses hold and use the superscript "0" generally to denote distributions, data, or statistics generated under the global null.

Define the statistic corresponding to the observed number of $\alpha$-level rejections as $\hat{\theta}=$ $\sum_{w=1}^{W} 1\left\{T_{w}>c_{w, \alpha}\right\}$. Its counterpart in a sample generated under the global null is $\hat{\theta}^{0}$ and in a resample $j$ is $\hat{\theta}^{(j)}$. Using $F$ to denote cumulative distribution functions (CDFs), respectively define the true CDF of the number of rejections under the global null, its counterpart in the resamples, and its empirical estimator in the resamples as:

$$
\begin{gathered}
F_{\hat{\theta}^{0}}(r)=P\left(\hat{\theta}^{0} \leq r\right) \\
F_{\hat{\theta}^{(j)}}(r)=P\left(\hat{\theta}^{(j)} \leq r\right)
\end{gathered}
$$




$$
\hat{F}_{\hat{\theta}^{(j)}}(r)=\frac{1}{B} \sum_{j^{*}=1}^{B} 1\left\{\hat{\theta}^{\left(j^{*}\right)} \leq r\right\}
$$

We denote almost sure convergence, convergence in probability, convergence in distribution, and ordinary limits respectively as " $\underset{N \rightarrow \infty}{\stackrel{A . S .}{\longrightarrow}}$ ", " $\underset{N \rightarrow \infty}{\stackrel{P}{\longrightarrow}}$ ", " $\underset{N \rightarrow \infty}{\stackrel{D}{\longrightarrow}}$ ", and " $\underset{N \rightarrow \infty}{\longrightarrow}$ ".

\section{MAIN RESULTS}

We now develop theory allowing us to approximate the sampling distribution of $F_{\hat{\theta}^{0}}$ through resampling. Specifically, we show that under a certain class of resampling algorithms defined below, the empirical sampling distribution of the number of rejections in the resamples converges to the true distribution of the number of rejections in samples generated under the global null. We chose to characterize the sampling distribution empirically rather than theoretically because it does not appear to have a tractable closed form without imposing assumptions on the correlation structure of the tests and potentially requiring asymptotics on the number of hypothesis tests. (Despite the intractable sampling distribution, it is straightforward to derive at least the exact variance of $\hat{\theta}^{0}$ if the pairwise correlations between the $p$-values are known; see the Supplement). Because simulation error associated with using a finite number of resamples to approximate the CDF of the resampled data can be made arbitrarily small by taking $B \rightarrow \infty$, we follow convention (e.g., Freedman (1981)) in ignoring this source of error and considering only asymptotics on $N$.

\subsection{An assumption on the resampling algorithm}

To establish the main convergence result, we will use the following key assumption stating that, regardless of whether the observed sample was generated under the global null or under an alternative, the resampling algorithm must generate a sampling distribution for $T^{(j)}$ that converges to the sampling distribution of $T^{0}$ (that is, in samples generated under the global null). We will later discuss resampling algorithms that satisfy this assumption.

Assumption 4.3. The resampling algorithm used to generate $Z^{(j)}$ must ensure that $T^{(j)} \underset{N \rightarrow \infty}{\stackrel{D}{\longrightarrow}}$ $T^{0}$, or equivalently $F_{T^{(j)}} \underset{N \rightarrow \infty}{\longrightarrow} F_{T^{0}}$.

Typically, resampling algorithms fulfilling this assumption will need to preserve the correlation structure of all variables in the dataset, except where the global null dictates otherwise. If not, the distribution of the test statistics will usually not be preserved. Additionally, just as the original data are assumed to respect the parametric assumptions of all $W$ hypothesis tests, the resampled data must be generated in a manner that also respects this parametric structure. Otherwise, hypothesis tests conducted on the resampled data may not preserve their nominal $\alpha$-levels, which again affects the distribution of the test statistics. 
Remark 4.1. For Assumption 4.3 to hold, it is sufficient for $T$ to be a continuous function

of $Z$ and for $Z^{(j)} \underset{N \rightarrow \infty}{\stackrel{D}{\longrightarrow}} Z^{0}$. Note that this condition is not necessary; for example, Westfall $\&$ Young (1993) propose several algorithms that induce the global null by centering the data themselves by sample estimates, rather than by centering the test statistics as in Algorithm 4.1 below. In such cases, Assumption 4.3 may hold without $Z^{(j)} \underset{N \rightarrow \infty}{\stackrel{D}{\longrightarrow}} Z^{0}$.

\subsection{Valid residual resampling for OLS}

We now consider the design of valid resampling algorithms for the familiar setting of ordinary least squares (OLS) multiple regression. Assume that each of $W$ outcome variables, $\left(Y_{1}, \cdots, Y_{W}\right)$, is regressed on the same design matrix, $X \in \mathbb{R}^{N \times p}$, comprising an intercept term denoted $X_{1}$ (such that the residuals have mean 0 ), a single exposure of interest (taken without loss of generality to be $\left.X_{2}\right)$, and the adjusted covariates $\left(X_{3}, \cdots, X_{C}\right)$. Assume all covariates besides the intercept are mean-centered. Thus, the dataset $Z$ contains a random vector $\left(1, X_{n 2}, \cdots, X_{n C}, Y_{n 1}, \cdots, Y_{n W}\right)$ for each subject $n$.

Let $\epsilon_{w}=\left(\epsilon_{1 w}, \cdots, \epsilon_{N w}\right)$ denote the $N$-vector of true errors for the $w^{\text {th }}$ regression such that $\epsilon_{n w} \sim N\left(0, \sigma_{w}^{2}\right)$. Let $\hat{\epsilon}_{w}$ be its estimated counterpart (the residuals). Let $\sigma_{w}^{2}=E\left[\epsilon_{n w}^{2} \mid X\right]$ as usual, and assume $\sigma_{w}^{2}<\infty$. Let $\widehat{\gamma}_{i w}$ be the $i^{\text {th }}$ coefficient estimate in the $w^{\text {th }}$ regression, and denote the coefficient for the exposure of interest in the $w^{\text {th }}$ regression model as $\widehat{\beta}_{w}=\widehat{\gamma}_{2 w}$. The $W$ models are then:

$$
\begin{gathered}
Y_{n 1}=\gamma_{11}+\beta_{1} X_{n 2}+\sum_{j=3}^{C} \gamma_{j 1} X_{n j}+\epsilon_{n 1} \\
\vdots \\
Y_{n W}=\gamma_{1 W}+\beta_{W} X_{n 2}+\sum_{j=3}^{C} \gamma_{j W} X_{n j}+\epsilon_{n W}
\end{gathered}
$$

Let $\beta^{W}=\left(\beta_{1}, \cdots, \beta_{W}\right)$ be a $W$-vector containing, for each regression model, the single coefficient of interest, and let $\hat{\beta}^{W}$ and $\hat{\beta}^{W(j)}$ denote its sample estimate in the original dataset and in resampled dataset $j$, respectively. Suppose without loss of generality that the null hypotheses of interest are $H_{0 w}: \beta_{w}=0$.

Letting hats denote the usual OLS estimates obtained from the original sample, the usual test statistics in the original sample are:

$$
T=\left(\frac{\hat{\beta}_{1}}{\hat{\sigma}_{1}\left(X^{\prime} X\right)^{-1 / 2}}, \cdots, \frac{\hat{\beta}_{W}}{\hat{\sigma}_{W}\left(X^{\prime} X\right)^{-1 / 2}}\right)
$$


Their unobservable counterparts centered to reflect the global null are:

$$
T^{0}=\left(\frac{\hat{\beta}_{1}-\beta_{1}}{\hat{\sigma}_{1}\left(X^{\prime} X\right)^{-1 / 2}}, \cdots, \frac{\hat{\beta}_{W}-\beta_{W}}{\hat{\sigma}_{W}\left(X^{\prime} X\right)^{-1 / 2}}\right)
$$

Algorithm 4.1 (A valid resampling algorithm for OLS). Generalizing from Freedman (1981)), a parametric resampling algorithm that satisfies Assumption 4.3 is to first fix the covariates $\left(X_{1}, \cdots, X_{C}\right)$ for all observations while setting the resampled "outcomes" equal to the fitted values plus a vector of residuals resampled with replacement. That is, letting $n^{\prime}$ denote an observation sampled with replacement, the resampled variables for observation $n$ are:

$$
\begin{array}{cc}
X_{n 1}^{(j)}:=X_{n 1} & Y_{n 1}^{(j)}:=\hat{Y}_{n 1}+\left(\hat{Y}_{n^{\prime} 1}-Y_{n^{\prime} 1}\right) \\
\vdots & \vdots \\
X_{n C}^{(j)}:=X_{n C} & Y_{n W}^{(j)}:=\hat{Y}_{n W}+\left(\hat{Y}_{n^{\prime} W}-Y_{n^{\prime} W}\right)
\end{array}
$$

Then each test statistic in the resamples is computed using $\widetilde{H}_{0 w}: \beta_{w}=\hat{\beta}_{w}$ in order to recover the null sampling distribution (Hall \& Wilson, 1991). That is:

$$
T^{(j)}=\left(\frac{\hat{\beta}_{1}^{(j)}-\hat{\beta}_{1}}{\hat{\sigma}_{1}^{(j)}\left(X^{\prime} X\right)^{-1 / 2}}, \cdots, \frac{\hat{\beta}_{W}^{(j)}-\hat{\beta}_{W}}{\hat{\sigma}_{W}^{(j)}\left(X^{\prime} X\right)^{-1 / 2}}\right)
$$

We show in the Supplement that this resampling algorithm fulfills Assumption 4.3 because the distribution of each $\hat{\beta}_{w}-\beta_{w}=\left(X^{\prime} X\right)^{-1} X^{\prime} \epsilon_{w}$ (in the original sample) depends only on the true error distribution and not on $\beta_{w}$, so the resampling algorithm need only recover the true error distribution to provide valid inference under the global null.

Various other approaches that may appear valid in fact violate the assumption. For example, we could fix the design matrix $X$ but resample with replacement the outcome vectors, $\left(Y_{n^{\prime} 1}, \cdots, Y_{n^{\prime} W}\right)$, rather than the residuals:

$$
\begin{array}{cc}
X_{n 1}^{(j)}:=X_{n 1} & Y_{n 1}^{(j)}:=Y_{n^{\prime} 1}^{(j)} \\
\vdots & \vdots \\
X_{n C}^{(j)}:=X_{n C} & Y_{n W}^{(j)}:=Y_{n^{\prime} W}^{(j)}
\end{array}
$$

Although this approach indeed enforces the global null and preserves the correlation between the outcomes, it fails to preserve the correlations between the outcomes and the adjusted covariates and thus does not recover the distribution of $T^{0}$.

A second incorrect alternative would be to bootstrap parametrically from Equation (1) while enforcing the global null by constraining each $\beta_{w}^{(j)}:=0$ :

$$
X_{n 1}^{(j)}:=X_{n 1} \quad Y_{n 1}:=\hat{\gamma}_{11}+\sum_{j=3}^{C} \hat{\gamma}_{j 1} X_{n j}+\epsilon_{n 1}
$$




$$
\begin{array}{cc}
\vdots & \vdots \\
X_{n C}^{(j)}:=X_{n C} & Y_{n W}:=\hat{\gamma}_{1 W}+\sum_{j=3}^{C} \hat{\gamma}_{j W} X_{n j}+\epsilon_{n W}
\end{array}
$$

where $\epsilon_{n w}^{(j)} \sim_{i . i . d .} N\left(0, \hat{\sigma}_{\epsilon_{n w}}^{2}\right)$. However, this sequential algorithm fails to entirely preserve the correlations among the outcomes if there are unmeasured variables, beyond the adjusted covariates, that contribute to these correlations. In turn, the distribution of $T^{0}$ is not recovered. A final incorrect alternative would be a generic bootstrap hypothesis test performed by resampling with replacement entire rows of data and then centering the test statistics as in Equation (2). However, this algorithm incorrectly treats the design matrix as random rather than fixed, which would be appropriate for correlation models but not the intended regression models (Freedman, 1981). Additionally, this algorithm can produce data violating the assumptions of standard OLS inference, even when the original data fulfill the assumptions. Suppose, for example, that the design matrix contains only an intercept and a binary exposure of interest, $X_{2} \in\{0,1\}$, and that, for some outcome $Y_{w^{*}}$, we have $\beta_{w^{*}} \neq 0$ (i.e., the alternative hypothesis holds). Then, of course, $\hat{\epsilon}_{n w^{*}}$ may be normal, allowing valid OLS inference, despite that $Y_{w^{*}}$ itself may be bimodal with peaks at $E\left[Y_{w^{*}} \mid X_{2}=0\right]$ and $E\left[Y_{w^{*}} \mid X_{2}=1\right]$. This generic resampling algorithm retains the bimodality of $Y_{w^{*}}$ while breaking the association between $Y_{w^{*}}$ and $X_{1}$; thus, the resampled residuals $\hat{\epsilon}_{n w^{*}}^{(j)}$ will be bimodal rather than normal (Westfall \& Young, 1993), and standard inference may fail.

\subsection{Valid inference on the number of rejections}

We now present the main theorem establishing that resampling algorithms fulfilling Assumption 4.3, such as Algorithm 4.1 for OLS, also yield valid inference on the number of rejections.

Theorem 4.1. Under Assumption 4.3. $\hat{\theta}^{(j)} \underset{N \rightarrow \infty}{\stackrel{D}{\longrightarrow}} \hat{\theta}^{0}$.

Proof. Define the $r$-family of "rejection sets" as all possible configurations of the $W$ test statistics that lead to $r$ rejections:

$$
\mathcal{A}_{r}=\left\{\left(A_{1}, \cdots, A_{W}\right) \in \mathbb{R}^{W}:\left(T_{1} \in A_{1}, \cdots, T_{W} \in A_{W}\right) \Rightarrow \hat{\theta}=r\right\}
$$

Consider the limiting distribution of $\hat{\theta}^{(j)}$ :

$$
\begin{aligned}
\lim _{N \rightarrow \infty} P\left(\hat{\theta}^{(j)}=r\right) & =\lim _{N \rightarrow \infty} P\left(\sum_{w=1}^{W} 1\left\{T_{w}^{(j)}>c_{w, \alpha}\right\}=r\right) \\
& =\lim _{N \rightarrow \infty} \sum_{\left(A_{1}, \cdots, A_{W}\right) \in \mathcal{A}} P\left(T_{1}^{(j)} \in A_{1}, \cdots, T_{W}^{(j)} \in A_{W}\right)
\end{aligned}
$$




$$
\begin{aligned}
& =\sum_{\left(A_{1}, \cdots, A_{W}\right) \in \mathcal{A}} P\left(T_{1}^{0} \in A_{1}, \cdots, T_{W}^{0} \in A_{W}\right) \\
& =P\left(\hat{\theta}^{0}=r\right)
\end{aligned}
$$

where the second equality follows from Assumption 4.3 .

To summarize, Theorem 4.1 implies that valid inference, including the null interval and global test, can be conducted using the distribution of the number of rejections in resamples generated using an algorithm fulfilling Assumption 4.3 .

\section{PRACTICAL USE AND INTERPRETATION}

In practice, to estimate the proposed metrics, one would first use a resampling algorithm fulfilling Assumption 4.3 to generate a large number of resamples under the global null (e.g., $B=1,000)$. Then, the lower and upper bounds of a $95 \%$ null interval can be defined as the $2.5^{\text {th }}$ and $97.5^{\text {th }}$ percentiles of $\left(\hat{\theta}^{(1)}, \cdots, \hat{\theta}^{(j)}\right)$, and the $p$-value for the global test is the empirical tail probability:

$$
P_{N}\left(\hat{\theta}^{(j)} \geq \hat{\theta}\right)=\frac{1}{B} \sum_{j^{*}=1}^{B} 1\left\{\hat{\theta}^{\left(j^{*}\right)} \geq \hat{\theta}\right\}
$$

We provide an $\mathrm{R}$ package, NRejections, to automate the resampling and estimation process for OLS models (see Supplement).

The $p$-value for the global test can be interpreted as the probability of observing at least $\hat{\theta}$ rejections in samples generated under the global null. The null interval can be interpreted as the plausible range of $\hat{\theta}$ in samples generated under the global null. The excess hits, computed as the difference between $\hat{\theta}$ and the upper limit of the null interval, can be interpreted as the number of rejections exceeding what would be expected in $95 \%$ of samples under the global null. Note that although the number of excess hits is not necessarily equivalent to the number of false null hypotheses (a point reiterated in the Discussion), such an interpretation is in fact valid (under repeated sampling at least $95 \%$ of time) for test statistics satisfying subset pivotality, as shown in the next result.

Definition 5.1 (Subset pivotality). Define the set of all tested null hypotheses as $\mathcal{W}$. The distribution of $\left(T_{1}, \cdots, T_{W}\right)$ fulfills subset pivotality if, for any $\mathcal{K} \subseteq \mathcal{W}$, the joint distribution of $\left\{T_{w}: w \in \mathcal{K}\right\}$ is independent of the truth or falsehood of $\left\{H_{0, w}: w \in \mathcal{W} \backslash \mathcal{K}\right\}$ (Westfall 6 Young, 1993).

Theorem 5.1. Let $\theta_{\text {hi }}$ denote the upper limit of a $95 \%$ null interval. Define the set of all tested true null hypotheses as $\mathcal{K}^{\prime} \subseteq \mathcal{W}=\left\{w: H_{0, w}\right.$ holds for exactly $\left.w \in \mathcal{K}^{\prime}\right\}$. Then, under subset pivotality, $\theta_{h i}$ can be interpreted as the maximum number of false positives among the observed rejections at least 95\% of the time under repeated sampling, and regardless of the configuration of true and false null hypotheses. That is: 


$$
P\left(\sum_{w \in \mathcal{K}^{\prime}} 1\left\{T_{w}>c_{w, \alpha}\right\}>\theta_{h i} \mid H_{0, w} \text { holds for exactly } w \in \mathcal{K}^{\prime}\right) \lesssim 0.05
$$

where $\lesssim$ denotes that the inequality holds asymptotically. Equivalently, 95\% of the time under repeated sampling, the number of false null hypotheses is as least the number of excess hits, $\hat{\theta}-\theta_{h i}$.

Proof. The probability of obtaining more than $f$ false positives across tests at level $\alpha$ is:

$$
P\left(\sum_{w \in \mathcal{K}^{\prime}} 1\left\{T_{w}>c_{w, \alpha}\right\}>f \mid H_{0, w} \text { holds for exactly } w \in \mathcal{K}^{\prime}\right)
$$

By subset pivotality, the conditioning statement can be expanded to include the hypotheses outside $\mathcal{K}^{\prime}$, such that the global null holds:

$$
=P\left(\sum_{w \in \mathcal{K}^{\prime}} 1\left\{T_{w}>c_{w, \alpha}\right\}>f \mid H_{0, w} \text { holds } \forall w \in \mathcal{W}\right)
$$

Conditional on the global null, we have $\mathcal{K}^{\prime}=\mathcal{W}$; hence:

$$
\begin{aligned}
& =P\left(\sum_{w \in \mathcal{W}} 1\left\{T_{w}>c_{w, \alpha}\right\}>f \mid H_{0, w} \text { holds } \forall w \in \mathcal{W}\right) \\
& =P\left(\hat{\theta}>f \mid H_{0, w} \text { holds } \forall w \in \mathcal{W}\right) \\
& =P\left(\hat{\theta}^{0}>f\right)
\end{aligned}
$$

Choosing $f=\theta_{h i}$, the above equality becomes:

$$
\begin{aligned}
P\left(\sum_{w \in \mathcal{K}^{\prime}} 1\left\{T_{w}>c_{w, \alpha}\right\}>\theta_{h i} \mid H_{0, w} \text { holds } \forall w \in \mathcal{K}^{\prime}\right) & =P\left(\hat{\theta}^{0}-\theta_{h i}>0\right) \\
& \lesssim 0.05
\end{aligned}
$$

The asymptotic bound holds by Theorem 4.1. The left-hand side represents the probability of obtaining more than $\theta_{h i}$ false positives under any configuration of true and false hypotheses, $\left(H_{0,1}, \cdots, H_{0, W}\right)$, because $\mathcal{K}^{\prime}$ is simply the arbitrary subset of $\mathcal{W}$ for which $H_{0, w}$ does hold. The right-hand side represents the probability of observing at least one excess hit.

Remark 5.1. Letting $\alpha_{W}$ denote an arbitrary level of global inference, a general $\left(1-\alpha_{W}\right) \%$ null interval has an analogous interpretation, again under subset pivotality. That is, its upper 
limit can be interpreted as the maximum number of false positives at least $\left(1-\alpha_{W}\right) \%$ of the time under repeated sampling. Equivalently, $\left(1-\alpha_{W}\right) \%$ of the time under repeated sampling, the number of false null hypotheses is at least the number of excess hits based on a $\left(1-\alpha_{W}\right) \%$ null interval. These interpretations hold regardless of $\alpha$, the level of the individual hypothesis tests: for example, a 99\% null interval constructed based on individual tests at $\alpha=0.05$ nevertheless leads to global inference on the number of false positives at the $\alpha_{W}=0.01$ level.

Subset pivotality holds for OLS and a number of other common choices of test statistics (Westfall \& Young, 1993), in which case the excess hits based on a 95\% null interval can thus be interpreted as the minimum number of false null hypotheses such that this statement would hold $95 \%$ of the time with repeated sampling. We further illustrate interpretation of the proposed metrics in the following applied example.

\section{APPLIED EXAMPLE}

Existing epidemiologic analyses have investigated the extent to which an individual's experience of parental warmth during childhood is associated with the individual's later "flourishing" in mid-life (Chen et al., 2019). Flourishing has been broadly conceived as a state of positive mental health comprising high emotional, psychological, and social well-being (Keyes, 2002), and reductive analyses that individually assess its theorized components, such as perceived purpose in life and positive affect, may not fully capture potential impacts of the overall experience of flourishing (Keyes \& Simoes, 2012; VanderWeele, 2017).

\subsection{Methods}

Closely reproducing Chen et al. (2019)'s methods, we conducted longitudinal analyses of a subset of $N=2,697$ subjects from the "Mid-life in the United States" (MIDUS) cohort study (Brim et al. 2004) of 7,108 adults, recruited to include siblings and twin pairs. For simplicity in these analyses, we randomly selected only one sibling from within each sibship. In an initial wave of data collection (1995-1996), subjects recalled the parental warmth that they experienced during childhood as an average of separate scales of maternal and paternal warmth. In a second wave (2004-2006), the same subjects reported 13 continuous subscales of flourishing in emotional, psychological, and social domains (Keyes, 2002).

We first reproduced Chen et al. (2019)'s main analysis by assessing the association between a one-unit increase in standardized parental warmth (i.e., an increase of one standard deviation on the raw scale) with a standardized, continuous composite measure of flourishing ("overall flourishing"), which aggregated the 13 subscales per Keyes (2002, 2007). We conducted similar analyses for the remaining 16 continuous outcome variables in Chen et al. (2019)'s analyses, namely the 3 standardized composite scores for each domain (emotional, psychological, and social) treated separately and the 13 individual subscales. All of our analyses controlled for age, sex, race, nativity status, parents' nativity status, number of siblings, and other 
childhood family factors. We expected the resulting 17 test statistics to be correlated both because of conceptual similarities between the subscale variables (e.g., social acceptance and social integration) and because of the composite and domain measures' direct arithmetic relationships with their subscales. Last, to characterize overall evidence strength across the 17 outcomes, we resampled per Algorithm 4.1 with $B=5,000$ to estimate the null interval and excess hits (with each test conducted at either $\alpha=0.05$ or $\alpha=0.01$ ) and to conduct the global test using the number of rejections in individual tests conducted at $\alpha=0.05$. All data and code required to reproduce these analyses is publicly available and documented (https://osf.io/qj9wa/).

\subsection{Results}

Table S1 (Supplement) displays demographics and childhood family characteristics in our sample, comprising all covariates adjusted in analysis. The 17 outcome measures had a median correlation magnitude of $|r|=0.39$ (minimum $=0.12$; maximum $=0.89 ; 25^{\text {th }}$ percentile $=0.28 ; 75^{\text {th }}$ percentile $=0.55$ ). The composite analysis estimated that, controlling for demographics and childhood family factors, individuals reporting an additional standard deviation (SD) of parental warmth in childhood experienced greater mid-life flourishing by, on average, $b=0.22$ (95\% CI: $[0.18,0.26])$ SDs.

Of the 17 outcomes considered individually, all were "significantly" associated with parental warmth at $\alpha=0.05$ (i.e., $\hat{\theta}=17$ ), and 15 were "significantly" associated at $\alpha=0.01$. The mean standardized effect size was $b=0.14$. The directions of all effects suggested that increased parental warmth was associated with improved flourishing outcomes (Table 2). In contrast, if parental warmth were in fact unassociated with any of the outcomes, we would expect $17 \times 0.05=0.85$ rejections with a null interval of $[0,5]$ at $\alpha=0.05$ (Figure 11). At $\alpha=0.01$, we would expect 0.17 rejections with null interval $[0,2]$ at $\alpha=0.01$. Thus, at $\alpha=0.05$ and $\alpha=0.01$ respectively, we observed $17-5=12$ and $15-2=13$ excess hits above what would be expected in $95 \%$ of samples under the global null. Thus, $95 \%$ of the time under repeated sampling, at least 13 of the 17 null hypotheses would be false, indicating nonzero associations of parental warmth. Indeed, a global test based on the number of rejections at $\alpha=0.05$ suggested very strong evidence against the global null ( $p=0$ because every resampled dataset had $<17$ rejections; Figure 1). (By comparison, simple inference based on the exact binomial distribution, assuming anticonservatively that the outcomes are independent, yields a too-narrow null interval at $\alpha=0.05$ of $[0,3]$ and a global $p$-value of $0.05^{17}=7 \times 10^{-23}$.) Overall, our composite analyses strongly support small effects of parental warmth on composite flourishing, as reported by Chen et al. (2019) (Table 2, first row); our novel analyses of $\hat{\theta}$ additionally provide compelling global evidence for associations of parental warmth with flourishing across the 17 outcomes, accounting for their correlation structure. 
Table 2: OLS estimate $(\hat{\beta})$ characterizing association of a 1-SD increase in parental warmth with each of 17 standardized flourishing outcomes, adjusting for all covariates in Table S1 (Supplement). Inference is not multiplicity-corrected.

\begin{tabular}{|c|c|c|}
\hline Outcome & $\hat{\beta}[95 \% \mathrm{CI}]$ & $p$-value \\
\hline \multicolumn{3}{|l|}{ Overall and domain composites } \\
\hline Overall flourishing & $0.22[0.18,0.26]$ & $<2 \times 10^{-16}$ \\
\hline Emotional well-being & $0.21[0.17,0.25]$ & $<2 \times 10^{-16}$ \\
\hline Social well-being & $0.13[0.08,0.17]$ & $2 \times 10^{-9}$ \\
\hline Psychological well-being & $0.20[0.16,0.24]$ & $<2 \times 10^{-16}$ \\
\hline \multicolumn{3}{|l|}{ Emotional well-being subscales } \\
\hline Positive affect & $0.19[0.15,0.23]$ & $<2 \times 10^{-16}$ \\
\hline Life satisfaction & $0.19[0.15,0.23]$ & $<2 \times 10^{-16}$ \\
\hline \multicolumn{3}{|l|}{ Social well-being subscales } \\
\hline Meaningfulness of society & $0.04[0,0.08]$ & 0.048 \\
\hline Social integration & $0.15[0.11,0.19]$ & $5 \times 10^{-13}$ \\
\hline Social acceptance & $0.09[0.05,0.13]$ & $3 \times 10^{-5}$ \\
\hline Social contribution & $0.09[0.05,0.13]$ & $1 \times 10^{-5}$ \\
\hline Social actualization & $0.06[0.02,0.11]$ & 0.002 \\
\hline \multicolumn{3}{|l|}{ Psychological well-being subscales } \\
\hline Autonomy & $0.08[0.04,0.12]$ & $3 \times 10^{-4}$ \\
\hline Environmental mastery & $0.14[0.09,0.18]$ & $6 \times 10^{-11}$ \\
\hline Personal growth & $0.11[0.07,0.15]$ & $4 \times 10^{-7}$ \\
\hline Positive relations & $0.25[0.21,0.29]$ & $<2 \times 10^{-16}$ \\
\hline Purpose in life & $0.05[0.01,0.09]$ & 0.018 \\
\hline Self-acceptance & $0.22[0.18,0.26]$ & $<2 \times 10^{-16}$ \\
\hline
\end{tabular}

\section{Simulation StUdy}

We conducted a simulation study with two objectives. First, we compared the estimated null interval to $\hat{\theta}$ for varying effect sizes in an outcome-wide study and to characterize how its precision depends on the strength of correlation between the hypothesis tests and on the $\alpha$ level used for each test. Second, we assessed the power of global tests conducted using the number of rejections with $\alpha=0.05$ or $\alpha=0.01$ for each individual test or derived from the five existing FWER-control methods listed in Table 1. As an additional measure of power, we compared the total number of rejected null hypotheses at a familywise-controlled $\alpha_{W}=0.05$ identified by each method (using the excess hits when considering our method per Theorem 5.1]. All code required to reproduce the simulation study is publicly available (https://osf.io/qj9wa/). 
Figure 1: Number of rejections $\left(\hat{\theta}^{(j)}\right)$ for each of 5,000 resamples. Solid lines: $E\left[\hat{\theta}^{0}\right]=\alpha \times 17$. Dashed lines: upper limit of 95\% null interval.

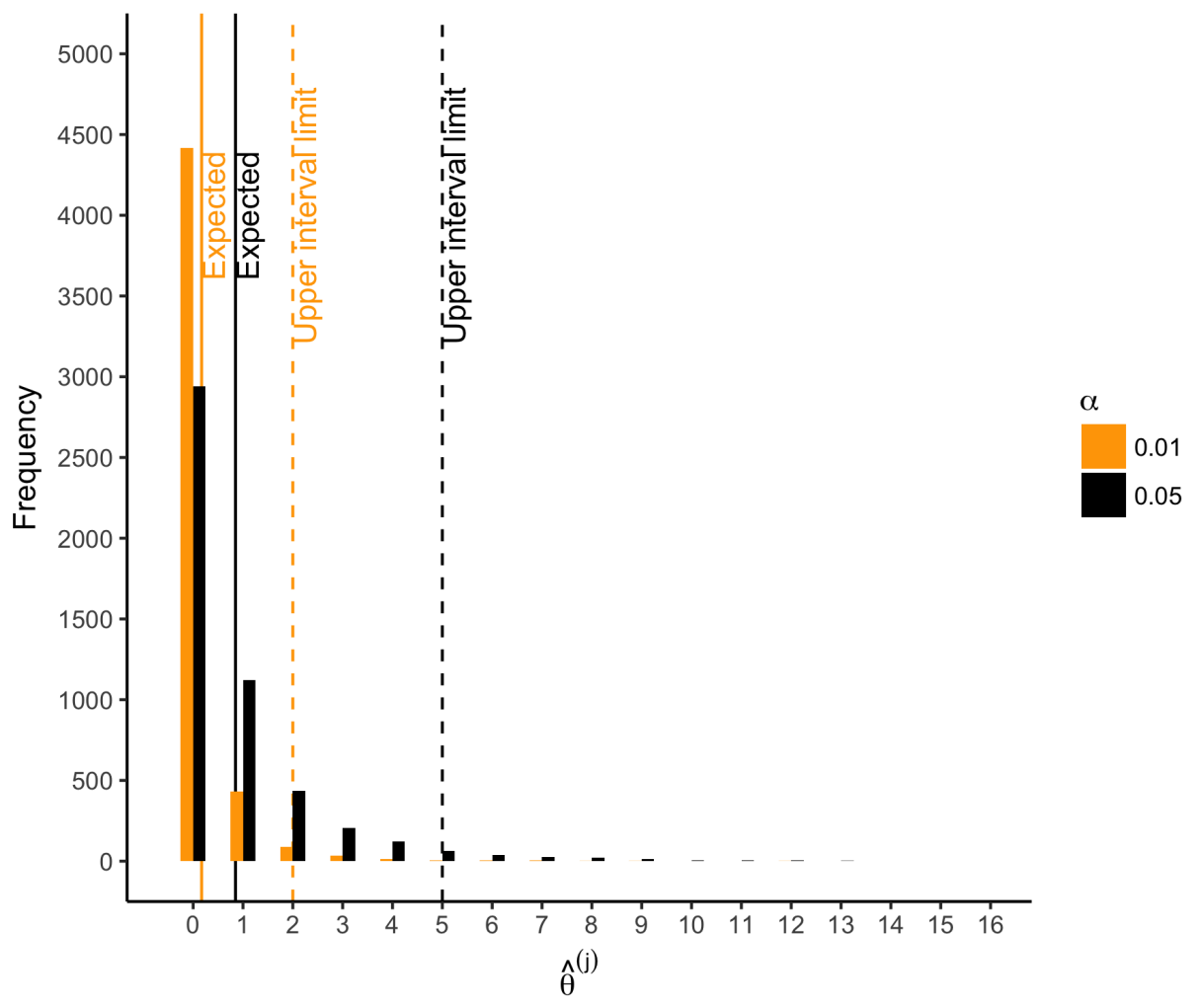

\subsection{Methods}

We generated multivariate standard normal data, comprising 1 covariate $(X)$ and 40 outcomes $\left(Y_{1}, \cdots, Y_{40}\right)$ for a fixed $N=1,000$ subjects. The correlation between each pair of outcomes was $\rho_{Y Y}$. The correlation between $X$ and a proportion, $q$, of outcomes was $\rho_{X Y}$, with remaining pairs having correlation 0 . We manipulated scenario parameters in a full-factorial design (Table 3). Each of 500 simulations per scenario proceeded as follows:

1. We generated an observed dataset according to the scenario.

2. We regressed each outcome $Y_{w}$ on $X$ and conducted a $t$-test at level $\alpha$ on the coefficient for $X$. We computed $\hat{\theta}$.

3. For each resampling iterate $j$ (with $B=1,000$ ), we resampled based on the algorithm in Algorithm 4.1. We conducted a $t$-test at level $\alpha$ on the coefficient for $X$ and computed $\hat{\theta}^{(j)}$.

4. We used the quantiles of $\left(\hat{\theta}^{(1)}, \cdots, \hat{\theta}^{(B)}\right)$ to construct the null interval, compute the excess hits, and conduct our proposed joint test. 
Table 3: Possible values of simulation parameters.

\begin{tabular}{llll}
\hline$\rho_{X Y}$ & $\rho_{Y Y}$ & $q$ & $\alpha$ \\
\hline 0.03 & 0 & 0 & 0.01 \\
0.05 & 0.10 & 0.20 & 0.05 \\
0.10 & 0.30 & 0.50 & \\
0.15 & 0.60 & 1 &
\end{tabular}

5. We used the $t$-statistics or $p$-values from the resamples to conduct joint tests based on the existing methods.

6. For FWER methods, we computed the number of multiplicity-corrected rejections to indicate the number of rejected null hypotheses at familywise $\alpha_{W}=0.05$. For our methods, per Theorem 5.1, we instead set the number of rejected null hypotheses equal to the maximum of 0 and the excess hits based on a $95 \%$ null interval.

(We resampled per Algorithm 4.1 for all resampling-based methods. However, Section 4.2.2 of Westfall \& Young (1993) suggests a different residual-resampling algorithm for OLS in which the resampled residuals alone are used as the resampled outcomes, such that $Y_{n w}^{(j)}:=\hat{Y}_{n^{\prime} w}-Y_{n^{\prime} w}$, where $n^{\prime}$ is a resampled observation. Thus, the global null is already enforced in the resampled data, and the test statistics do not require centering. Because the truth or falsehood of each null hypothesis changes the sampling distribution of the OLS coefficient estimates only by a location shift and the subset pivotality assumption described in Section 2 holds for OLS (Westfall \& Young, 1993, Section 4.2.2), the difference between this algorithm and the one we used is immaterial, as confirmed by additional simulations that are not shown.)

\subsection{Results}

Figure 2 displays $\hat{\theta}$ in samples generated under the global null (row 4, panel 1) or under varying alternatives, as well as mean limits of 95\% null intervals. (For simplicity, Figure 2 does not show all scenarios, but rather excludes some smaller effect sizes. For comprehensive results, see the Supplement.) As expected for a resampling algorithm fulfilling Assumption 4.3. the null intervals appeared identical regardless of whether the data were generated under the global null. As the pairwise correlation strength between outcomes increased, the null intervals became substantially less precise. For example, with tests conducted at $\alpha=0.05$, the mean upper limit of the null interval was nearly three times as high for $\rho_{Y Y}=0.60$ versus $\rho_{Y Y}=0$ (i.e., 14.9 versus 5.0 rejections; see the leftmost and rightmost null intervals within each panel). Thus, with a true effect size of $\rho_{X Y}=0.05$ for all pairs (Figure 2, row 1, panel 3), the mean number of observed rejections at $\alpha=0.05$ (i.e., 13.8) would be within the $95 \%$ null interval if the outcomes had correlation strength of $\rho_{Y Y}=0.60$ (excess hits $=13.8-14.9=-0.8$ ), but would be well outside the null interval, and thus provide 
stronger evidence for global association, if the outcomes were independent (excess hits $=$ $13.8-5.0=8.8$.

Figures 3 and 4 respectively show the power of each global test and the number of rejected null hypotheses. (Again, we show a subset of scenarios, excluding those in which all methods had nearly $100 \%$ power and excluding some intermediate correlation strengths. These scenarios differ from those in Figure 2. Comprehensive results appear in the Supplement.) As expected, when data were generated under the global null, all methods had approximately nominal or conservative false positive rates (Figure 3, row 3, panel 1). Our proposed global test achieved its best performance with weakly correlated or independent statistics (Figure 3. left sides of each panel) and when a moderate to high proportion of alternative hypotheses were true $(q>0.20)$. In such scenarios, our proposed methods sometimes rejected as many as 6.0 more null hypotheses on average than the next-best method (Figure 4 , row 2, panel 3). In contrast, the power of our methods declined when few alternative hypotheses were true (e.g., $q=0.20$; Figure 3 , rows 1-2, panels 1 ), likely because in these scenarios, $\hat{\theta}$ would often have been near its expectation under the global null. Simultaneously, the small number of $p$-values corresponding to the true alternative hypotheses may often have been quite small, improving the power of tests derived from FWER methods. Interestingly, in all scenarios we considered, Romano \& Wolf (2007)'s method uniformly outperformed methods other than the one we propose; it also outperformed ours with highly correlated test statistics, but not always with weakly correlated or independent statistics. 
Figure 2: $95 \%$ null intervals versus mean rejections in observed datasets ( $\times$ ). Panels: Null and alternative data-generating mechanisms of original samples. Points and error bars: Mean $\hat{\theta}^{(j)}$ and mean limits of null intervals with tests at $\alpha=0.01$ (yellow) or at $\alpha=0.05$ (red).
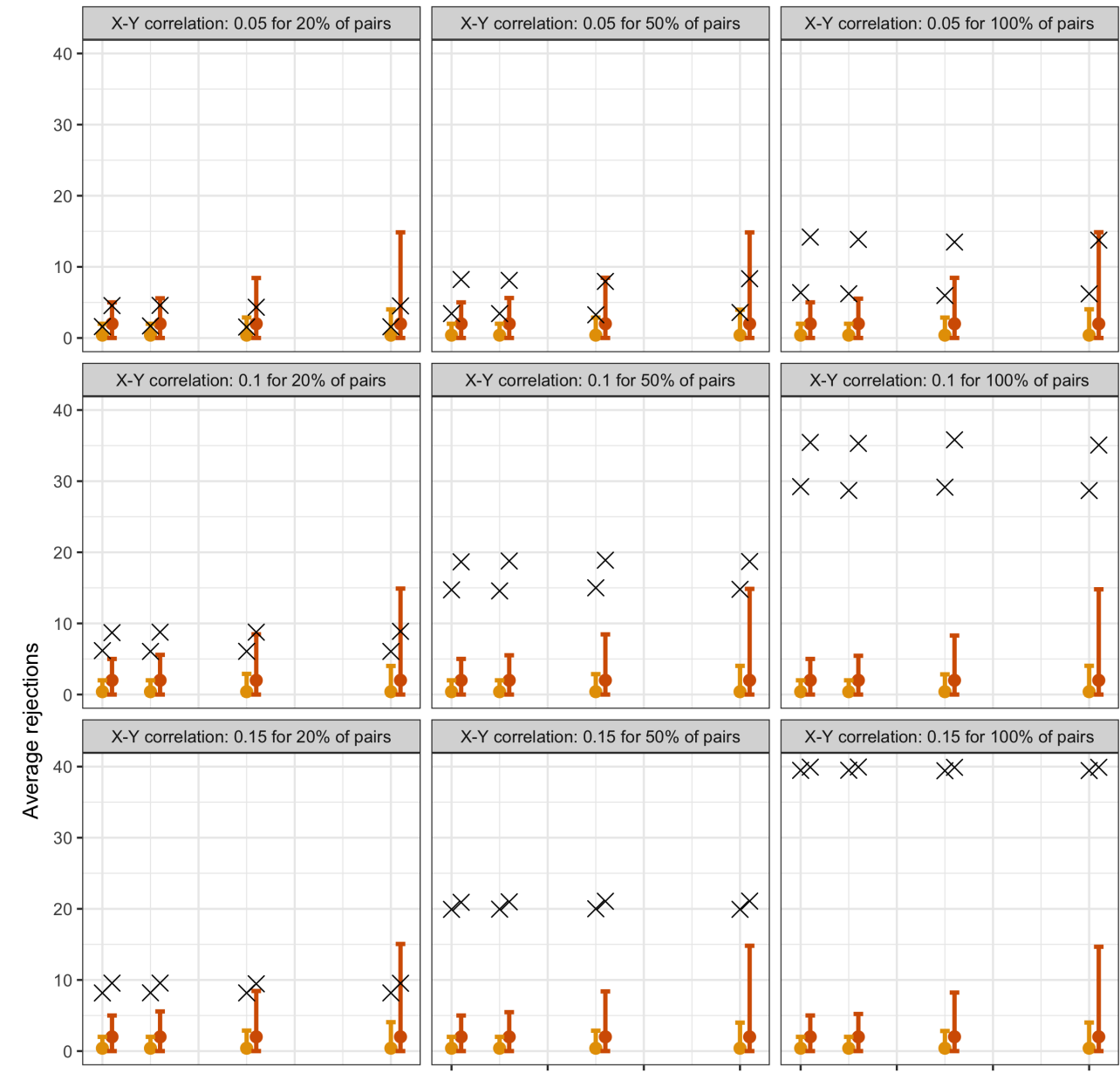

X-Y correlation: 0.1 for $100 \%$ of pairs
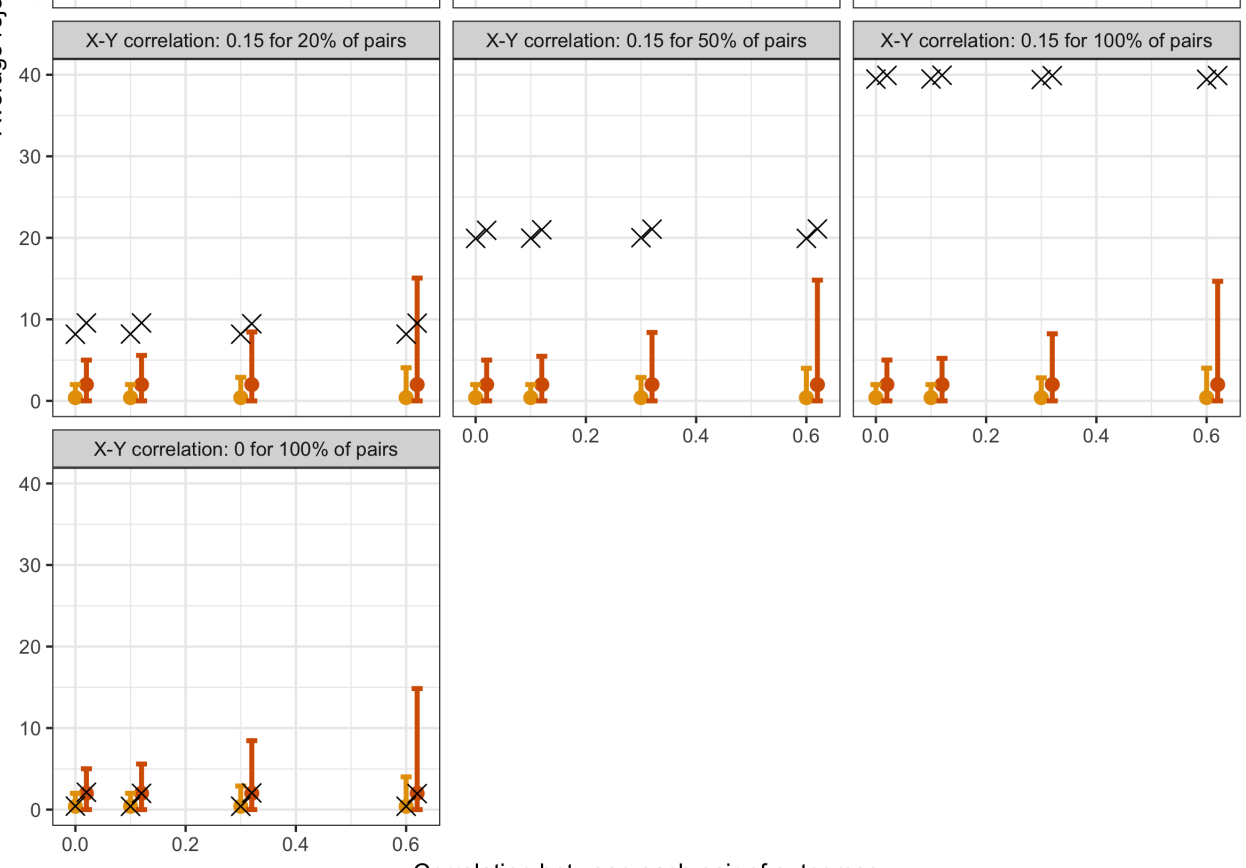

Correlation between each pair of outcomes

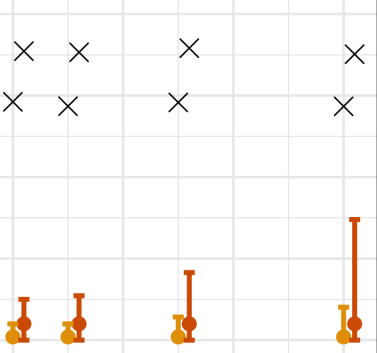


Figure 3: Power of global tests based on existing FWER-control procedures and on the number of rejections. $B=$ Bonferroni, $H=H o l m, M P=\min P, W S=$ Wstep, $R=$ Romano, $G 1=$ number of rejections at $\alpha=0.01, G 5=$ number of rejections at $\alpha=0.05$.
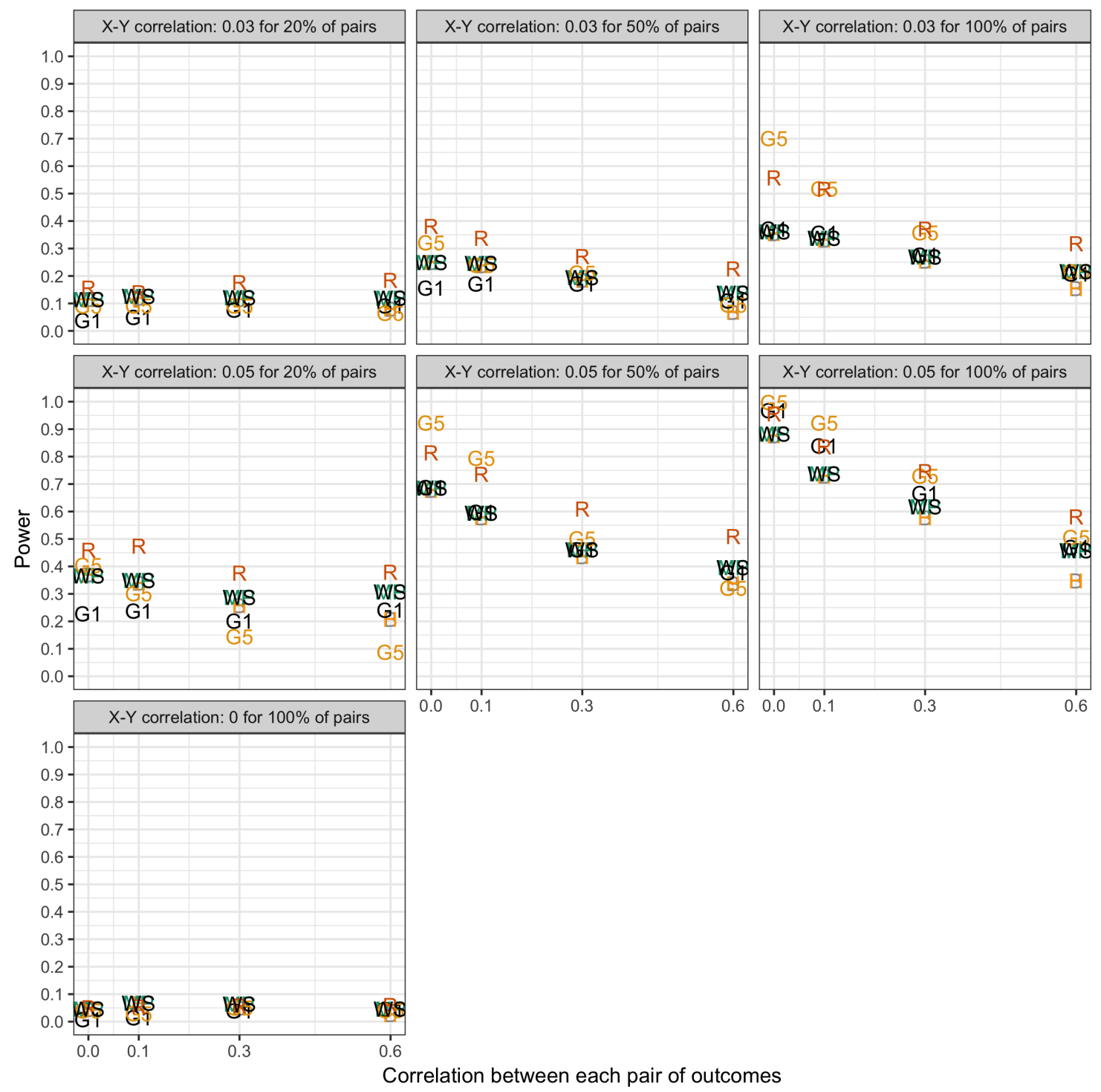
Figure 4: Number of rejected null hypotheses at familywise-controlled $\alpha_{W}=0.05$ based on existing $F W E R$-control procedures and on the excess hits. $B=$ Bonferroni, $H=H o l m, M P=\operatorname{minP}$, $W S=$ Wstep,$R=$ Romano, $G 1=$ number of rejections at $\alpha=0.01$, G5=number of rejections at $\alpha=0.05$.

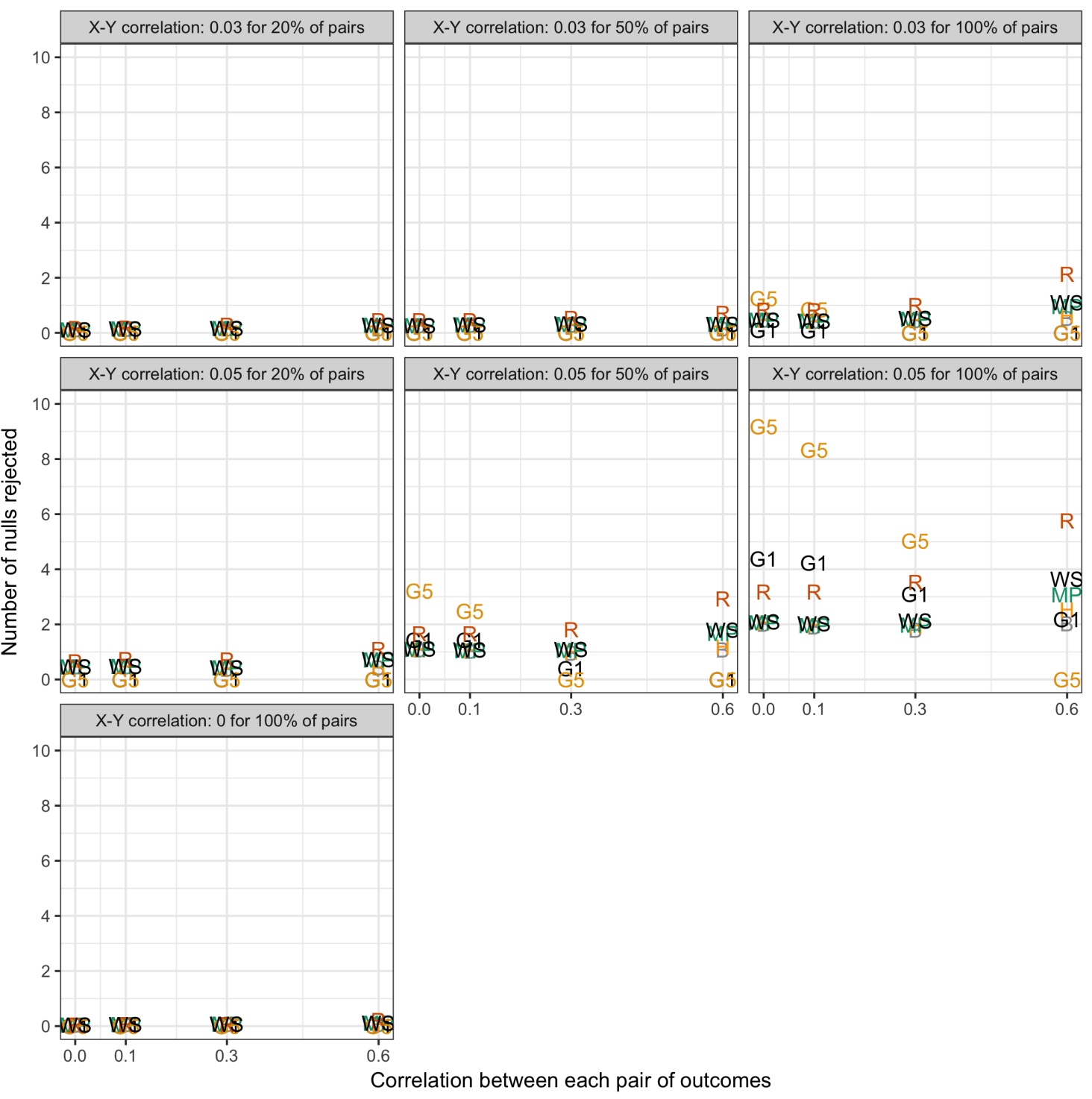

Besides Romano \& Wolf (2007)'s method, the other existing methods, even the conservative naïve methods, performed comparably to one another (within approximately 10 percentage points of power of one another for nearly all scenarios). Based on simple additional simulations (Supplement, Figure S4), we speculate that this somewhat counterintuitive finding arises because the methods appear to differ primarily in their degree of adjustment for those $p$-values that are $\gg 0.05$, with the resampling-based methods typically yielding substantially smaller, but still "nonsignificant", adjusted values for these large $p$-values. In contrast, $p$-values near the 0.05 threshold - those that could potentially affect results of the global test - appear to receive only small and comparable adjustments across all methods. Thus, we speculate that 
it is rather unlikely that a sample would have all adjusted $p$-values above 0.05 under a naïve approach, but would have at least one $p$-value adjusted to below 0.05 under a resampling approach.

\section{Discussion}

This paper has characterized global evidence strength across arbitrarily correlated hypothesis tests without being restricted to the setting of high-dimensional analyses. Specifically, we proposed metrics that compare the observed number of test rejections, $\hat{\theta}$, to its expected sampling distribution under the global null. $\hat{\theta}$ is a simple summary measure that seems of natural interest; the proposed metrics help to rigorously ground intuition regarding its behavior when tests are correlated. First, we proposed reporting a null interval for the number of $\alpha$-level rejections expected in $95 \%$ of samples generated under the global null along with the number of excess hits observed above the upper interval limit. Second, we proposed reporting a one-sided test of the global null whose $p$-value represents the probability of observing at least $\hat{\theta}$ rejections in samples generated under the global null. For OLS models, these metrics can be easily estimated via resampling using our R package, NRejections.

Existing methods that control FWER for arbitrarily correlated tests can also be used to conduct such a global test, so we conducted a simulation study assessing their relative power. To our knowledge, this is the first direct comparison of these methods as global tests, rather than as FWER-control procedures. All methods showed nominal or conservative false positive rates, as expected theoretically. Our method performed well when tests were independent or weakly correlated and when a moderate to high proportion of alternative hypotheses were true; therefore, it may be most suitable for studies in which the uncorrected $p$-values are relatively similar to one another, rather than for studies in which a small number of uncorrected $p$-values are much smaller than the others. The global test based on Romano \& Wolf (2007)'s method performed very well overall and, in the OLS scenarios we considered, appeared to uniformly outperform existing methods other than sometimes our own, concerning which Romano \& Wolf (2007)'s method was often more powerful, though ours sometimes performed better with weakly correlated or independent tests. In these contexts, considering the excess hits sometimes allowed rejection of many more null hypotheses with familywise control than the next-best method (e.g., an average of 9.17 vs. 3.17 in one such scenario).

We speculate that when Romano \& Wolf (2007)'s method has superior power to ours as a global test, despite its additional need to strongly control FWER, this reflects the loss of information inherent in dichotomizing $p$-values at $\alpha$ to compute the number of rejections. A more powerful global test might be based, for example, on departures of the observed joint ECDF of the $p$-values, treated as continuous, from their CDF under the global null, as estimated via resampling methods such as those outlined in this paper and in Westfall \& Young (1993). However, even in contexts in which a global test derived from Romano \& Wolf (2007)'s method does provide better power (though this is not always the case), the null 
interval and excess hits may still be of interest. More broadly, we view $\hat{\theta}$ and the proposed metrics as useful summaries of global evidence strength that do lose some information in the process of summarization. As such, they supplement, rather than replace, reporting individual, continuous $p$-values with and without standard multiplicity corrections.

Our consideration of existing methods has focused on repurposing those that adjust individual $p$-values or critical values. Other existing methods, like our proposed metrics, do directly characterize overall evidence strength and merit some discussion. For example, global inference on regression coefficients for different outcomes can be conducted using multivariate regression (Johnson \& Wichern, 2002) or Zellner (1962)'s "seemingly unrelated regressions" generalization. However, these approaches only modestly improve efficiency compared to that achieved in $W$ separate OLS models, and when the design matrix is shared across models, coefficient estimates are identical to those in OLS models (Oliveira \& Teixeira-Pinto, 2015). Another approach to global inference is to meta-analyze the effect sizes from each analysis (Cumming, 2014, Goh et al. 2016, Shaffer, 1995). Compared to direct analysis of the raw data, meta-analysis is likely to be inefficient. Alternatively, one could conduct global inference on a reduced number of outcomes by constructing composite measures (as in the applied example) or applying statistical dimension reduction, such as principal components analysis, though some information is lost. Another class of methods conducts global inference through the direct combination of $p$-values, though these methods almost always assume independence or a known parametric dependence structure (Benjamini \& Heller (2008); Brown (1975); Fisher (1948); Kost \& McDermott (2002); Whitlock (2005); Zaykin et al. (2002); but see Poole et al. (2016) for a nonparametric exception) or apply only for specific types of genetic data (Aerts et al., 2006).

When interpreting our proposed metrics, it is important to note that they characterize the sampling distribution under, specifically, the global null. Thus, rejecting the global test at $\alpha=0.05$ indicates that there is no more than a $5 \%$ probability of observing at least $\hat{\theta}$ rejections in samples generated under the global null. The excess hits can be interpreted as the minimum number of false null hypotheses $95 \%$ of the time under repeated sampling only under subset pivotality and otherwise must not be misinterpreted as such. Statements to this effect can be also made using procedures that strongly control FWER. By construction, these procedures ensure that for a familywise $\alpha_{W}=0.05$, in $95 \%$ of samples generated under any configuration of null and alternative hypotheses, each rejected test will represent a true positive; therefore, the number of rejections based on inference adjusted to strongly control FWER can also be interpreted as the minimum number of false null hypotheses $95 \%$ of the time under repeated sampling. There are also interesting methods designed specifically for providing confidence statements regarding the number of false null hypotheses Goeman et al. 2011). Because these methods require resampling under various intersections of the null hypotheses, they appear well-suited to hypothesis tests that span multiple exposures, for which intersection testing can simply involve testing a single regression model on all the exposures against various nested null models. However, these methods appear less suitable to hypothesis tests spanning multiple outcomes, for which intersection testing is 
more challenging.

Our proposed methods are valid for arbitrary hypothesis tests as long as data are resampled such that the resampled test statistics converge in distribution to the distribution they would have under the global null. To this end, an additional contribution of this paper is the theoretical justification of residual resampling for OLS models in the context of multiple testing, informed by Freedman (1981)'s work for a single regression model and Westfall \& Young (1993)'s related algorithms. Indeed, a central challenge for resampling-based methods for multiple testing in general is the design of valid resampling procedures. The present theory supports using residual resampling under the global null for OLS in the context of our methods, of FWER control (Romano \& Wolf, 2005a, 2007, Westfall \& Young, 1993), of FDP control (Yekutieli \& Benjamini, 1999), and of corrections for "data snooping" (Romano $\&$ Wolf 2005b). We focused on OLS-based hypothesis tests because of their generality and ability to subsume many common tests. However, for certain other tests, such as those based on GLMs with non-identity link functions, validly resampling under the global null appears to be an open problem, although algorithms have been developed outside the multiple testing context for confidence intervals (e.g., Moulton \& Zeger (1991)) and, under additional assumptions, for permutation hypothesis tests (Potter, 2005). Other estimators, such as those using propensity score matching, pose challenges for resampling because the estimators lack certain smoothness properties; these challenges arise even without the need to enforce the global null (Abadie \& Imbens, 2008). Algorithms fulfilling Assumption 4.3 for such estimators could potentially use subsampling to relax some of the smoothness assumptions of with-replacement resampling (Politis \& Romano, 1994).

Correlated test statistics can naturally arise not only when testing multiple associations between exposures and outcomes, but also when multiple hypothesis tests are used to investigate the same question, as in "data snooping" (Romano \& Wolf, 2005b). For example, investigators often fit several regression models to investigate the same association of interest, adjusting for different sets of covariates or using different subsets of the data. Situating these "researcher degrees of freedom" (Simmons et al., 2011) within a formal multiple testing context (Romano \& Wolf, 2005b), rather than merely reporting a single result chosen post hoc, could help reduce unnecessary false positives in the literature and may additionally foster a more balanced overall view of the evidence. Our proposed metrics provide one approach to summarizing evidence in such settings; for example, the $p$-value from the global test could help characterize evidence supporting a false null hypothesis in at least one of the multiple model specifications.

In summary, the number of rejections across correlated hypothesis tests can be a useful summary measure of overall evidence strength when reported with metrics such as a null interval, the number of excess hits, and a test of the global null. Reporting these metrics alongside $p$-values with and without standard multiplicity corrections may provide a richer view of global evidence strength than corrected inference alone. 


\section{REPRODUCIBILITY}

All code required to reproduce the applied example and simulation study is publicly available (https://osf.io/qj9wa/). The dataset used for the applied example is publicly available through the Inter-University Consortium for Political and Social Research (ICPSR); we detail how to access the dataset and reproduce the applied example in our public repository (https://osf.io/u69s2/).

\section{ACKNOWLEDGMENTS}

We thank Tom Chen for helpful comments on the manuscript and Ying Chen for providing analysis code for the applied example. This research was supported by National Institutes of Health grants R01 CA222147, R01 LM013866, UL1TR003142, P30CA124435, and P30DK116074. The MIDUS study was funded by the John D. and Catherine T. MacArthur Foundation Research Network and the National Institute on Aging (P01-AG020166 and U19-AG051426). The funders had no role in the design, conduct, or reporting of this research. 


\section{REFERENCES}

Abadie, A., \& Imbens, G. W. (2008). On the failure of the bootstrap for matching estimators. Econometrica, 76 (6), 1537-1557.

Aerts, S., Lambrechts, D., Maity, S., Van Loo, P., Coessens, B., De Smet, F., ... others (2006). Gene prioritization through genomic data fusion. Nature Biotechnology, 24(5), 537.

Benjamini, Y., \& Heller, R. (2008). Screening for partial conjunction hypotheses. Biometrics, $64(4), 1215-1222$.

Blakesley, R. E., Mazumdar, S., Dew, M. A., Houck, P. R., Tang, G., Reynolds III, C. F., \& Butters, M. A. (2009). Comparisons of methods for multiple hypothesis testing in neuropsychological research. Neuropsychology, 23(2), 255.

Brim, O. G., Ryff, C. D., \& Kessler, R. C. (2004). The MIDUS national survey: An overview. University of Chicago Press.

Brown, M. B. (1975). A method for combining non-independent, one-sided tests of significance. Biometrics, 987-992.

Chen, Y., Kubzansky, L. D., \& VanderWeele, T. J. (2019). Parental warmth and flourishing in mid-life. Social Science \& $\mathcal{S}^{2}$ Medicine, 220, 65-72.

Clarke, S., Hall, P., et al. (2009). Robustness of multiple testing procedures against dependence. The Annals of Statistics, 37(1), 332-358.

Cumming, G. (2014). The new statistics: Why and how. Psychological Science, 25(1), 7-29.

Dunn, O. J. (1961). Multiple comparisons among means. Journal of the American Statistical Association, 56(293), 52-64.

Fisher, R. A. (1948). Combining independent tests of significance. American Statistician, $2(5), 30$.

Freedman, D. A. (1981). Bootstrapping regression models. The Annals of Statistics, $1218-1228$.

Friguet, C., Kloareg, M., \& Causeur, D. (2009). A factor model approach to multiple testing under dependence. Journal of the American Statistical Association, 104(488), 1406-1415.

Goeman, J. J., Solari, A., et al. (2011). Multiple testing for exploratory research. Statistical Science, 26(4), 584-597. 
Goh, J. X., Hall, J. A., \& Rosenthal, R. (2016). Mini meta-analysis of your own studies: Some arguments on why and a primer on how. Social and Personality Psychology Compass, $10(10), 535-549$.

Hall, P., \& Wilson, S. R. (1991). Two guidelines for bootstrap hypothesis testing. Biometrics, $757-762$.

Holm, S. (1979). A simple sequentially rejective multiple test procedure. Scandinavian Journal of Statistics, 65-70.

Johnson, R. A., \& Wichern, D. (2002). Multivariate analysis. Wiley Online Library.

Keyes, C. L. (2002). The mental health continuum: From languishing to flourishing in life. Journal of Health and Social Behavior, 207-222.

Keyes, C. L. (2007). Promoting and protecting mental health as flourishing: A complementary strategy for improving national mental health. American Psychologist, 62(2), 95.

Keyes, C. L., \& Simoes, E. J. (2012). To flourish or not: Positive mental health and all-cause mortality. American Journal of Public Health, 102(11), 2164-2172.

Kost, J. T., \& McDermott, M. P. (2002). Combining dependent p-values. Statistics \& Probability Letters, 60(2), 183-190.

Leek, J. T., \& Storey, J. D. (2008). A general framework for multiple testing dependence. Proceedings of the National Academy of Sciences, 105(48), 18718-18723.

Moulton, L. H., \& Zeger, S. L. (1991). Bootstrapping generalized linear models. Computational statistics \& data analysis, 11, 53-63.

Oliveira, R., \& Teixeira-Pinto, A. (2015). Analyzing multiple outcomes: Is it really worth the use of multivariate linear regression? Journal of Biometrics 83 Biostatistics, 6 .

Politis, D. N., \& Romano, J. P. (1994). Large-sample confidence regions based on subsamples under minimal assumptions. The Annals of Statistics, 2031-2050.

Poole, W., Gibbs, D. L., Shmulevich, I., Bernard, B., \& Knijnenburg, T. A. (2016). Combining dependent p-values with an empirical adaptation of Brown's method. Bioinformatics, 32(17), i430-i436.

Potter, D. M. (2005). A permutation test for inference in logistic regression with small-and moderate-sized data sets. Statistics in Medicine, 24(5), 693-708.

Romano, J. P., \& Wolf, M. (2005a). Exact and approximate stepdown methods for multiple hypothesis testing. Journal of the American Statistical Association, 100(469), 94-108. 
Romano, J. P., \& Wolf, M. (2005b). Stepwise multiple testing as formalized data snooping. Econometrica, $73(4), 1237-1282$.

Romano, J. P., \& Wolf, M. (2007). Control of generalized error rates in multiple testing. The Annals of Statistics, 1378-1408.

Shaffer, J. P. (1986). Modified sequentially rejective multiple test procedures. Journal of the American Statistical Association, 81(395), 826-831.

Shaffer, J. P. (1995). Multiple hypothesis testing. Annual Review of Psychology, 46(1), $561-584$.

Simes, R. J. (1986). An improved Bonferroni procedure for multiple tests of significance. Biometrika, 73(3), 751-754.

Simmons, J. P., Nelson, L. D., \& Simonsohn, U. (2011). False-positive psychology: Undisclosed flexibility in data collection and analysis allows presenting anything as significant. Psychological Science, 22(11), 1359-1366.

Sun, W., \& Cai, T. (2009). Large-scale multiple testing under dependence. Journal of the Royal Statistical Society: Series B (Statistical Methodology), 71(2), 393-424.

van der Laan, M. J., Birkner, M. D., \& Hubbard, A. E. (2005). Empirical Bayes and resampling based multiple testing procedure controlling tail probability of the proportion of false positives. Statistical Applications in Genetics and Molecular Biology, 4(1).

van der Laan, M. J., Dudoit, S., \& Pollard, K. S. (2004). Augmentation procedures for control of the generalized family-wise error rate and tail probabilities for the proportion of false positives. Statistical Applications in Genetics and Molecular Biology, 3(1), 1-25.

VanderWeele, T. J. (2017). Outcome-wide epidemiology. Epidemiology, 28(3), 399-402.

VanderWeele, T. J., Mathur, M. B., \& Chen, Y. (2020). Outcome-wide longitudinal designs for causal inference: a new template for empirical studies. Statistical Science, 35(3), $437-466$.

Westfall, P. H., \& Troendle, J. F. (2008). Multiple testing with minimal assumptions. Biometrical Journal, 50(5), 745-755.

Westfall, P. H., \& Young, S. S. (1993). Resampling-based multiple testing: Examples and methods for p-value adjustment. Taylor \& Francis Group.

Whitlock, M. C. (2005). Combining probability from independent tests: the weighted Z-method is superior to Fisher's approach. Journal of Evolutionary Biology, 18(5), 13681373. 
Yekutieli, D., \& Benjamini, Y. (1999). Resampling-based false discovery rate controlling multiple test procedures for correlated test statistics. Journal of Statistical Planning and Inference, 82(1-2), 171-196.

Zaykin, D. V., Zhivotovsky, L. A., Westfall, P. H., \& Weir, B. S. (2002). Truncated product method for combining p-values. Genetic Epidemiology: The Official Publication of the International Genetic Epidemiology Society, 22(2), 170-185.

Zellner, A. (1962). An efficient method of estimating seemingly unrelated regressions and tests for aggregation bias. Journal of the American Statistical Association, 57(298), 348-368. 


\section{Supplement: New Metrics for Multiple Testing with Correlated Outcomes}

\section{EXACT VARIANCE UNDER GLOBAL NULL}

Let $p_{w}^{0}$ be the $p$-value in the $w^{t h}$ test under the global null, treated as a random variable. Then we have:

$$
\begin{aligned}
\operatorname{Var}\left(\widehat{\theta}^{0}\right) & =\operatorname{Var}\left(\sum_{w=1}^{W} 1\left\{p_{w}^{0}<\alpha\right\}\right) \\
& =\sum_{w=1}^{W} \operatorname{Var}\left(1\left\{p_{w}^{0}<\alpha\right\}\right)+2 \sum_{1 \leq i<j \leq W} \operatorname{Cov}\left(1\left\{p_{i}^{0}<\alpha\right\}, 1\left\{p_{j}^{0}<\alpha\right\}\right) \\
& =W \alpha(1-\alpha)+2 \sum_{1 \leq i<j \leq W} E\left[1\left\{p_{i}^{0}<\alpha, p_{j}^{0}<\alpha\right\}\right] \\
& -E\left[1\left\{p_{i}^{0}<\alpha\right\}\right] E\left[1\left\{p_{j}^{0}<\alpha\right\}\right] \\
& =W \alpha(1-\alpha)+2 \sum_{1 \leq i<j \leq W}\left[P\left(p_{i}^{0}<\alpha, p_{j}^{0}<\alpha\right)-\alpha^{2}\right]
\end{aligned}
$$

\section{Justifichtion of Algorithm 4.1}

In Theorem 2.3 below, we show that Algorithm 4.1 satisfies Assumption 4.3. The development of the proof is structured as follows. We make a regularity assumption (Assumption 2.1) and define how we will metrize convergence of the resampled test statistics (Definition 2.1). We bound the distance metric for certain types of random vectors (Lemma 2.1), in turn allowing us to bound the distance between the estimated sampling distribution in the resamples and the true sampling distribution to which the former should converge (Theorem 2.1). Using the latter bound, a triangle inequality argument, and convergence results regarding each term of the triangle inequality (Lemmas 2.2 and 2.3), we show the needed convergence result for the coefficient estimates (Theorem 2.2) and finally for the test statistics (Theorem 2.3).

First, assume the following regularity condition on the design matrix, which will later be relevant for the convergence of the coefficient estimates:

Assumption 2.1 (Regularity condition). Suppose without loss of generality that the regression covariate of interest is $X_{2}$. Correspondingly, let $B \in \mathbb{R}^{N \times 1}$ be the transposed second row of $\left(X^{\prime} X\right)^{-1} X^{\prime}$, or equivalently the first column of $X\left(X^{\prime} X\right)^{-1}$. (More generally, if the covariate 
of interest is the $i^{\text {th }}$ variable in the design matrix, then $B$ is defined as the $i^{\text {th }}$ row or column.) Assume that for some constant $k>0$ :

$$
\begin{aligned}
& N \cdot B^{\prime} B \underset{N \rightarrow \infty}{\stackrel{P}{\longrightarrow}} k \\
\Leftrightarrow & N \sum_{n=1}^{N}\left[\left(X^{\prime} X\right)^{-1} X^{\prime}\right]_{2 n}^{2} \underset{N \rightarrow \infty}{\stackrel{P}{\longrightarrow} k}
\end{aligned}
$$

where $\left[\left(X^{\prime} X\right)^{-1} X^{\prime}\right]_{2 n}$ denotes a matrix entry.

This assumption holds under the following sufficient conditions. Heuristically, these state that, for each regression model, the asymptotic standard errors of all $p$ regression coefficients are finite (A1), that the covariates have finite expectations and are not completely collinear (A2), and that the regression model does not fit perfectly (A3).

Proposition 2.1 (Sufficient conditions). Let $\mathcal{I}_{i j}$ denote an entry of the expected Fisher information matrix for an individual observation in the $w^{\text {th }}$ regression. Then Assumption 2.1 holds if, for all w:

$$
\begin{aligned}
& \mathcal{I}_{i i}>0 \forall i \in\{1, \cdots, p\} \\
& E\left[X_{n i} X_{n j}\right]<\infty \forall i, j \in\{1, \cdots, p\} \\
& \sigma_{w}^{2}>0
\end{aligned}
$$

Proof of Proposition 2.1. Let $\widehat{\gamma}_{i w}$ be the $i^{\text {th }}$ coefficient estimate in the $w^{\text {th }}$ regression, such that $\widehat{\gamma}_{2 w}=\widehat{\beta}_{w}$, the estimate of interest. Thus, let $\widehat{\alpha}_{w}=\left[\widehat{\gamma}_{1 w}, \widehat{\beta}_{w}, \widehat{\gamma}_{3 w}, \cdots, \widehat{\gamma}_{p w}\right]^{\prime}$ be the $p$-vector of estimates in the $w^{t h}$ regression. Denote a pairwise covariance $\operatorname{Cov}_{i j}=\operatorname{Cov}\left(\widehat{\gamma}_{i w}, \widehat{\gamma}_{j w}\right)$, and similarly denote a pairwise correlation as $\rho_{i j}$. Then the estimated covariance of $\widehat{\beta}_{w}$ with $\widehat{\gamma}_{i w}$ is:

$$
\begin{aligned}
\widehat{\operatorname{Cov}}_{2 i} & =\widehat{\rho}_{2 i} \cdot \widehat{\mathrm{SE}}\left(\widehat{\beta}_{w}\right) \cdot \widehat{\mathrm{SE}}\left(\widehat{\gamma}_{i w}\right) \\
& =\widehat{\rho}_{2 i} \cdot \frac{1}{\sqrt{N \widehat{\mathcal{I}}_{22}}} \cdot \frac{1}{\sqrt{N \widehat{\mathcal{I}}_{i i}}}
\end{aligned}
$$

With the left-hand side of Assumption 2.1 in view, we have:

$$
\left(X^{\prime} X\right)^{-1}=\frac{1}{\widehat{\sigma}_{w}^{2}}\left[\begin{array}{ccc}
\widehat{\operatorname{Cov}}_{11} & \cdots & \widehat{\operatorname{Cov}}_{1 p} \\
\widehat{\operatorname{Cov}}_{21} & \cdots & \widehat{\operatorname{Cov}}_{2 p} \\
\vdots & & \vdots \\
\widehat{\operatorname{Cov}}_{p 1} & \cdots & \widehat{\operatorname{Cov}}_{p p}
\end{array}\right]
$$




$$
\begin{aligned}
{\left[\left(X^{\prime} X\right)^{-1} X^{\prime}\right]_{2 n} } & =\frac{1}{\widehat{\sigma}_{w}^{2}}\left[\widehat{\operatorname{Cov}}_{21} \cdots \widehat{\operatorname{Cov}}_{2 p}\right]\left[X_{n 1} \cdots X_{n p}\right]^{\prime} \\
N \sum_{n=1}^{N}\left[\left(X^{\prime} X\right)^{-1} X^{\prime}\right]_{2 n}^{2} & =N \sum_{n=1}^{N}\left(\sum_{i=1}^{p} \frac{1}{\widehat{\sigma}_{w}^{2}} \widehat{\operatorname{Cov}}_{2 i} X_{n i}\right)^{2} \\
& =N \frac{1}{\widehat{\sigma}_{w}^{4}} \sum_{n=1}^{N}\left(\sum_{i=1}^{p} \sum_{j=1}^{p} \widehat{\operatorname{Cov}}_{2 i} \widehat{\operatorname{Cov}}_{2 j} X_{n i} X_{n j}\right) \\
& =N \frac{1}{\widehat{\sigma}_{w}^{4}} \sum_{i=1}^{p} \sum_{j=1}^{p} \widehat{\operatorname{Cov}}_{2 i} \widehat{\operatorname{Cov}}_{2 j} \sum_{n=1}^{N} X_{n i} X_{n j}
\end{aligned}
$$

Applying Equation (1) yields:

$$
\begin{aligned}
& =\frac{1}{\widehat{\sigma}_{w}^{4}} \sum_{i=1}^{p} \sum_{j=1}^{p} \widehat{\rho}_{2 i} \widehat{\rho}_{2 j} \frac{1}{\widehat{\mathcal{I}}_{22} \sqrt{\widehat{\mathcal{I}}_{i i} \widehat{\mathcal{I}}_{j j}}} \cdot \frac{1}{N} \sum_{n=1}^{N} X_{n i} X_{n j} \\
& \underset{N \rightarrow \infty}{\stackrel{P}{\longrightarrow}} \frac{1}{\sigma_{w}^{4}} \sum_{i=1}^{p} \sum_{j=1}^{p} \rho_{2 i} \rho_{2 j} \frac{1}{\mathcal{I}_{22} \sqrt{\mathcal{I}_{i i} \mathcal{I}_{j j}}} E\left[X_{n i} X_{n j}\right]
\end{aligned}
$$

If the sufficient conditions (A1)-(A3) above are fulfilled, this is a finite constant, as required.

We will consider the validity of the bootstrap in terms of convergence on the MallowsWasserstein metric, a conventional choice that is defined as follows (DasGupta, 2008, Freedman, 1981).

Definition 2.1 (Mallows-Wasserstein metric). Let $G_{A}$ and $G_{B}$ be arbitrary marginal distribution functions for random vectors $A \in \mathbb{R}^{W}$ and $B \in \mathbb{R}^{W}$, respectively. Then a form of Mallows-Wasserstein distance between $G_{A}$ and $G_{B}$ is the infimum, taken over all possible joint distributions for $(A, B)$ such that $A \sim G_{A}$ and $B \sim G_{B}$ marginally, of the expected $L_{2}$ distance between $A$ and $B$ :

$$
d_{2}\left(G_{A}, G_{B}\right):=\inf _{\substack{A \sim G_{A} \\ B \sim G_{B}}} E\left[\|A-B\|^{2}\right]^{1 / 2}
$$

We proceed to prove that the residual-resampling bootstrap is consistent with respect to the Mallows-Wasserstein metric in a development roughly following those of Freedman (1981) and Bickel \& Freedman (1981), who considered the asymptotic validity of residual resampling in recovering the sampling distribution of a $p$-vector of coefficient estimates from a single multiple linear regression model. Here, we extend this work to consider the sampling 
distribution of $\widehat{\beta}^{W}$. We first establish a lemma bounding the Mallows-Wasserstein distance between the distributions of two random vectors constructed as products of different random matrices with a single fixed vector.

Lemma 2.1. Let $C^{*}$ and $D^{*} \in \mathbb{R}^{W \times N}$ be random matrices from a specific joint distribution, and let $B \in \mathbb{R}^{N \times 1}$ be a fixed vector. Let $G_{C}$ and $G_{D}$ be the resulting marginal distribution functions of the vectors $C^{*} B$ and $D^{*} B \in \mathbb{R}^{W \times 1}$, respectively. Then:

$$
d_{2}\left(G_{C}, G_{D}\right)^{2} \leq \operatorname{tr}\left\{B B^{\prime} \cdot E\left[\left(C^{*}-D^{*}\right)^{\prime}\left(C^{*}-D^{*}\right)\right]\right\}
$$

Proof. First note that $d_{2}\left(G_{C}, G_{D}\right)^{2}$ is the infimum of the expectation over all possible joint distributions with marginals $G_{C}$ and $G_{D}$, whereas the quantity $E\left[\left\|C^{*} B-D^{*} B\right\|^{2}\right]$ is the expectation for a particular such joint distribution (i.e., the one giving rise to $C^{*} B$ and $D^{*} B$ ). We therefore have the inequality:

$$
\begin{aligned}
d_{2}\left(G_{C}, G_{D}\right)^{2} & \leq E\left[\left\|C^{*} B-D^{*} B\right\|^{2}\right] \\
& =E\left[\operatorname{tr}\{\underbrace{\left(C^{*} B-D^{*} B\right)}_{W \times 1} \underbrace{\left(C^{*} B-D^{*} B\right)^{\prime}}_{1 \times W}\}\right] \\
& =E\left[\operatorname{tr}\{\underbrace{\left(C^{*}-D^{*}\right)}_{W \times N} \underbrace{B B^{\prime}}_{N \times N} \underbrace{\left(C^{*}-D^{*}\right)^{\prime}}_{N \times W}\}\right] \\
& =E\left[\operatorname{tr}\{\underbrace{B B^{\prime}\left(C^{*}-D^{*}\right)^{\prime}\left(C^{*}-D^{*}\right)}_{N \times N}\}\right] \\
& =\operatorname{tr}\left\{E\left[B B^{\prime}\left(C^{*}-D^{*}\right)^{\prime}\left(C^{*}-D^{*}\right)\right]\right\} \\
& =\operatorname{tr}\left\{B B^{\prime} \cdot E[\underbrace{\left(C^{*}-D^{*}\right)^{\prime}\left(C^{*}-D^{*}\right)}_{N \times N}]\right\}
\end{aligned}
$$

The next theorem bounds the distance between the true sampling distribution of the estimated coefficients and the estimated sampling distribution in the resamples in terms of the distance between the sampling distribution of the true errors and the resampled residuals.

Theorem 2.1. Let $F$ denote the distribution function of the true errors for the $W$ regression models, $\left(\epsilon_{n 1}, \cdots, \epsilon_{n W}\right)$, and let $\widehat{F}_{N}$ denote the empirical distribution function of the residuals, which is used to approximate $F$ in Algorithm 4.1. Let $\Psi(F)$ denote the distribution of the standardized coefficient estimates, $\sqrt{N}\left(\widehat{\beta}^{W}-\beta^{W}\right)$, that are constructed as a function of the true error distribution; $\Psi(F)$ therefore represents the true sampling distribution to which a valid bootstrapped sampling distribution must converge. In contrast, let $\Psi\left(\widehat{F}_{N}\right)$ be the distribution of the standardized coefficient estimates in the resamples, $\sqrt{N}\left(\widehat{\beta}^{W(j)}-\widehat{\beta}^{W}\right)$, in which the empirical distribution of the residuals is used to approximate the true distribution. 
As in Assumption 2.1. let $B \in \mathbb{R}^{N \times 1}$ be the transposed second row of $\left(X^{\prime} X\right)^{-1} X^{\prime}$. Then:

$$
d_{2}\left(\Psi(F), \Psi\left(\widehat{F}_{N}\right)\right)^{2} \leq N \cdot \operatorname{tr}\left\{B B^{\prime}\right\} \cdot d_{2}\left(F, \widehat{F}_{N}\right)^{2}
$$

Proof. Let $U_{w}^{\prime} \in \mathbb{R}^{1 \times N}=\left[U_{1 w}, \cdots, U_{N w}\right]$ such that $\left(U_{n 1}, \cdots, U_{n W}\right) \sim F$ and:

$$
C \in \mathbb{R}^{W \times N}=\left[\begin{array}{cc}
- & U_{1}^{\prime} \\
\vdots \\
- & U_{W}^{\prime}-
\end{array}\right]=\left[\begin{array}{ccc}
U_{11} & \ldots & U_{N 1} \\
U_{12} & \ldots & U_{N 2} \\
\vdots & & \vdots \\
U_{1 W} & \ldots & U_{N W}
\end{array}\right]
$$

In general for multiple regression, we have $\widehat{\beta}-\beta=\left(X^{\prime} X\right)^{-1} X^{\prime} \epsilon$. Thus, we can express $\Psi(F)$ as the distribution of the $W$-vector:

$$
\sqrt{N}\left(\widehat{\beta}^{W}-\beta^{W}\right)=\sqrt{N}\left[\begin{array}{c}
{\left[\left(X^{\prime} X\right)^{-1} X^{\prime} U_{1}\right]_{2}} \\
\vdots \\
{\left[\left(X^{\prime} X\right)^{-1} X^{\prime} U_{W}\right]_{2}}
\end{array}\right]=\sqrt{N}\left[\begin{array}{c}
U_{1}^{\prime} B \\
\vdots \\
U_{W}^{\prime} B
\end{array}\right]=\sqrt{N} \cdot C B
$$

whose $w^{\text {th }}$ element pertains to the regression coefficient for $X_{2}$ in the $w^{\text {th }}$ regression. Let $D$ be the counterpart of $C$ with $\left(\widehat{U}_{n 1}, \cdots, \widehat{U}_{n W}\right) \sim \widehat{F}_{N}$ in place of $\left(U_{n 1}, \cdots, U_{n W}\right)$.

In view of Lemma 2.1. note that the entries of the matrix $(C-D)^{\prime}(C-D) \in \mathbb{R}^{N \times N}$ are:

$$
\begin{aligned}
{\left[(C-D)^{\prime}(C-D)\right]_{k j} } & =\sum_{w=1}^{W}\left[(C-D)^{\prime}\right]_{k w}[C-D]_{w j} \\
& =\sum_{w=1}^{W}[C-D]_{w k}[C-D]_{w j} \\
& =\sum_{w=1}^{W}\left(U_{k w}-\widehat{U}_{k w}\right)\left(U_{j w}-\widehat{U}_{j w}\right)
\end{aligned}
$$

We have $E\left[\left(U_{k w}-\widehat{U}_{k w}\right)\left(U_{j w}-\widehat{U}_{j w}\right)\right]=\operatorname{Cov}\left(U_{k w}-\widehat{U}_{k w}, U_{j w}-\widehat{U}_{j w}\right)$, but for all $k \neq j$, the covariance is 0 because the observations are independent. Thus, letting $I^{N}$ denote the $N \times N$ identity matrix, we have that $E\left[(C-D)^{\prime}(C-D)\right]$ is a diagonal matrix such that:

$$
E\left[(C-D)^{\prime}(C-D)\right]=I^{N} \cdot E\left[\sum_{w=1}^{W}\left(U_{j w}-\widehat{U}_{j w}\right)^{2}\right]
$$

which holds for any observation $j$ because they are identically distributed. In order to apply Lemma 2.1, we now restrict attention to a special choice of $C$ and $D$. First note that, by 
definition:

$$
d_{2}\left(F, \widehat{F}_{N}\right)^{2}=\inf _{\substack{\left(U_{j 1}, \cdots, U_{j W}\right) \sim F \\\left(\widehat{U}_{j 1}, \cdots, \widehat{U}_{j W}\right) \sim \widehat{F}_{N}}} E\left[\sum_{w=1}^{W}\left(U_{j w}-\widehat{U}_{j w}\right)^{2}\right]
$$

Now let $C^{*} \in \mathbb{R}^{W \times N}$ and $D^{*} \in \mathbb{R}^{W \times N}$ be a pair of random matrices constructed using random vectors $\left(U_{j 1}, \cdots, U_{j W}\right)$ and $\left(\widehat{U}_{j 1}, \cdots, \widehat{U}_{j W}\right)$ that follow the infimum-attaining joint distribution in Equation (3); that is, such that:

$$
E\left[\left(C^{*}-D^{*}\right)^{\prime}\left(C^{*}-D^{*}\right)\right]=I^{N} \cdot d_{2}\left(F, \widehat{F}_{N}\right)^{2}
$$

per the representations in Equations (2) and (3). (Such a choice exists by Bickel \& Freedman (1981)'s Lemma 8.1.) The result then follows immediately from applying Lemma 2.1, setting $G_{C}=\Psi(F), G_{D}=\Psi\left(\widehat{F}_{N}\right)$, and $B, C^{*}$, and $D^{*}$ as defined above and pulling the scalar $\sqrt{N}$ outside the squared distance.

Next, to apply the bound in Theorem 2.1, we will first bound the term on the right-hand side using a triangle inequality, which applies because $d_{2}(\cdot, \cdot)$ is a metric (Bickel \& Freedman, 1981). To this end, let $F_{N}$ denote the unobserved empirical distribution function of the true error vector, $\epsilon^{W}$. Then we have the following triangle inequality:

$$
d_{2}\left(\widehat{F}_{N}, F\right) \leq d_{2}\left(\widehat{F}_{N}, F_{N}\right)+d_{2}\left(F_{N}, F\right)
$$

The first term on the right-hand side relates the empirical distribution of the residuals to the empirical distribution of the true errors (which are both discrete distributions taking $N$ values); the second term relates the latter empirical distribution to the true error distribution (which is continuous). The next two lemmas bound the terms on the right-hand side of Equation (4); we will later use them to bound the left-hand side.

Lemma 2.2. For the expectation of the first term on the right-hand side of Equation (4):

$$
E\left[d_{2}\left(\widehat{F}_{N}, F_{N}\right)\right] \underset{N \rightarrow \infty}{\longrightarrow} 0
$$

Proof. As in Definition 2.1, let $U \sim \widehat{F}_{N}$ and $V \sim F_{N}$ be arbitrary random variables in $\mathbb{R}^{W}$ that follow the empirical marginal distributions of the residuals and of the true errors. Denote their elements $\left(U_{1}, \cdots, U_{W}\right)$ and $\left(V_{1}, \cdots, V_{W}\right)$. Let $\left(U^{*}, V^{*}\right)$ be the special choice of $(U, V)$ that follow not only the marginal empirical distributions $\widehat{F}_{N}$ and $F_{N}$, but also the empirical joint distribution of the residuals and the true errors. Then: 


$$
\begin{aligned}
d_{2}\left(\widehat{F}_{N}, F_{N}\right)^{2} & :=\inf _{\substack{U \sim \widehat{F}_{N} \\
V \sim F_{N}}} E\left[\|U-V\|^{2}\right] \\
& \leq E\left[\left\|U^{*}-V^{*}\right\|^{2}\right]
\end{aligned}
$$

because $\left(U^{*}, V^{*}\right)$ represents a choice of a single element from the set over which the infimum is taken. Expressing the right-hand side as the expectation of the joint empirical cumulative distribution function:

$$
\begin{aligned}
& =\frac{1}{N} \sum_{n=1}^{N} \underbrace{\sum_{w=1}^{W}\left(\widehat{\epsilon}_{n w}-\epsilon_{n w}\right)^{2}}_{\|\cdot\|^{2} \text { of a } W \text {-vector }} \\
& =\frac{1}{N} \sum_{w=1}^{W} \underbrace{\sum_{n=1}^{N}\left(\widehat{\epsilon}_{n w}-\epsilon_{n w}\right)^{2}}_{\|\cdot\|^{2} \text { of an } N \text {-vector }} \\
& =\frac{1}{N} \sum_{w=1}^{W}\left\|\widehat{\epsilon}_{w}-\epsilon_{w}\right\|^{2}
\end{aligned}
$$

The interchange of summations is used to express $W$ norms involving residuals from different regressions, summed over $N$ observations, as $N$ norms involving residuals of observations within a regression, summed over $W$ regressions. Taking expectations and using Freedman (1981)'s Eq. (2.2), this implies:

$$
\begin{aligned}
E\left[d_{2}\left(\widehat{F}_{N}, F_{N}\right)^{2}\right] & =\frac{p}{N} \sum_{w=1}^{W} \sigma_{w}^{2} \\
& \underset{N \rightarrow \infty}{\longrightarrow} 0
\end{aligned}
$$

By Jensen's inequality:

$$
E\left[d_{2}\left(\widehat{F}_{N}, F_{N}\right)\right] \underset{N \rightarrow \infty}{\longrightarrow} 0
$$


Lemma 2.3. Regarding the the second term on the right-hand side of Equation (4), we have:

$$
d_{2}\left(F_{N}, F\right) \underset{N \rightarrow \infty}{\stackrel{P}{\longrightarrow}} 0
$$

Proof. Letting $P_{N}$ denote an empirical probability, $F_{N}$ can be expressed as:

$$
\begin{aligned}
P_{N}\left(\epsilon_{n 1} \leq c_{1}, \cdots, \epsilon_{n W} \leq c_{W}\right) & =\frac{1}{N} \sum_{n=1}^{N} 1\left\{\epsilon_{n 1} \leq c_{1}, \cdots, \epsilon_{n W} \leq c_{W}\right\} \\
& \underset{N \rightarrow \infty}{A . S .} P\left(\epsilon_{n 1} \leq c_{1}, \cdots, \epsilon_{n W} \leq c_{W}\right)
\end{aligned}
$$

with the last line following from the Strong Law of Large Numbers (SLLN). Thus, $F_{N} \underset{N \rightarrow \infty}{\stackrel{A . S .}{\longrightarrow}} F$. Also by the SLLN, $\int\|x\|^{p} F_{N}(d x) \underset{N \rightarrow \infty}{\stackrel{A . S .}{\longrightarrow}} \int\|x\|^{p} F(d x)$ because the left-hand side is a sample average whereas the right-hand side is its expectation. These two results immediately imply Condition (a) of Bickel \& Freedman (1981)'s Lemma 8.3, which yields $d_{2}\left(F_{N}, F\right)^{2} \underset{N \rightarrow \infty}{\stackrel{P}{\longrightarrow} 0}$ and hence the desired result.

Theorem 2.2. The residual bootstrap is weakly consistent under the Mallows-Wasserstein metric for the OLS coefficient estimates (Definition 29.2 of DasGupta (2008)); that is:

$$
d_{2}\left(\Psi(F), \Psi\left(\widehat{F}_{N}\right)\right) \underset{N \rightarrow \infty}{\stackrel{P}{\longrightarrow}} 0
$$

Proof. Combining Theorem 2.1 with the triangle inequality in Equation (4) and observing that $\operatorname{tr}\left\{B B^{\prime}\right\}=\sum_{n=1}^{N} B_{N}^{2} \geq 0$ yields:

$$
d_{2}\left(\Psi(F), \Psi\left(\widehat{F}_{N}\right)\right) \leq \sqrt{N \cdot \operatorname{tr}\left\{B B^{\prime}\right\}} \cdot\left(d_{2}\left(\widehat{F}_{N}, F_{N}\right)+d_{2}\left(F_{N}, F\right)\right)
$$

The term $\sqrt{N \cdot \operatorname{tr}\left\{B B^{\prime}\right\}} \underset{N \rightarrow \infty}{\stackrel{P}{\longrightarrow}} k$ by Assumption 2.1 because $B B^{\prime}$ is scalar. By Markov's inequality, the convergence in mean of Lemma 2.2 implies that $d_{2}\left(\widehat{F}_{N}, F_{N}\right) \underset{N \rightarrow \infty}{\longrightarrow} 0$. Last, by Lemma 2.3. $d_{2}\left(F_{N}, F\right) \underset{N \rightarrow \infty}{\stackrel{P}{\longrightarrow}} 0$, so the desired result holds.

The next theorem uses the above result regarding convergence of the resampling-based coefficient estimates to establish convergence of the test statistics.

Theorem 2.3. Algorithm 4.1 fulfills Assumption 4.3; namely:

$$
T^{(j)} \underset{N \rightarrow \infty}{\stackrel{D}{\longrightarrow}} T^{0}
$$

Proof. By Bickel \& Freedman (1981)'s Lemma 8.3, Theorem 2.2 implies that

$$
\sqrt{N}\left(\widehat{\beta}^{W(j)}-\widehat{\beta}^{W}\right) \underset{N \rightarrow \infty}{\stackrel{D}{\longrightarrow}} \sqrt{N}\left(\widehat{\beta}^{W}-\beta^{W}\right)
$$


By Freedman (1981)'s Theorem 2.2, each $\widehat{\sigma}_{w}^{(j)} \underset{N \rightarrow \infty}{\stackrel{P}{\longrightarrow}} \sigma_{w}$. The desired result then follows from the multivariate Slutsky's Theorem. 


\section{APPLIED EXAMPLE}

Table 1 displays demographic and childhood family characteristics of the analyzed sample.

Table 1: Demographic and childhood family characteristics of 2,697 analyzed subjects. ${ }^{a}:$ By subject's adolescence, subject's family had ever been on welfare. ${ }^{b}$ : Ranged from 1 ("a lot better off" than others) to 7 ("a lot worse off" than others). ${ }^{c}$ : By age 16, subject had ever lived with an alcoholic.

\begin{tabular}{ll}
\hline Characteristic & Mean (SD) or \% \\
\hline Age & $46.89(12.35)$ \\
Female & $53.7 \%$ \\
Race & \\
$\quad$ White & $93.3 \%$ \\
$\quad$ Black & $3.6 \%$ \\
$\quad$ Other & $3.2 \%$ \\
Born in US & $95.8 \%$ \\
Mother born in US & $90.5 \%$ \\
Father born in US & $90.2 \%$ \\
Lived with biological parents & $81.1 \%$ \\
Number of siblings & $2.92(1.57)$ \\
Highest parental education & \\
$\quad$ Less than high school & $25.8 \%$ \\
$\quad$ High school & $36.0 \%$ \\
$\quad$ Some college & $15.8 \%$ \\
$\quad$ College degree or more & $22.5 \%$ \\
Childhood welfare & $5.6 \%$ \\
Subjective SES & \\
Residential area & $4.07(1.29)$ \\
$\quad$ Rural & \\
Small town & $23.1 \%$ \\
$\quad$ Medium town & $25.6 \%$ \\
$\quad$ Suburbs & $12.1 \%$ \\
$\quad$ City & $16.8 \%$ \\
Moved around & $18.3 \%$ \\
Residentially stable & $4.1 \%$ \\
Mother smoked & $74.1 \%$ \\
Father smoked & $32.6 \%$ \\
Lived with alcoholics ${ }^{c}$ & $62.0 \%$ \\
Importance of religion & $20.9 \%$ \\
\hline \multicolumn{2}{l}{ Continued on next page } \\
&
\end{tabular}


Table 1 - continued

\begin{tabular}{cl}
\hline Characteristic & Mean (SD) or \% \\
\hline Very important & $43.5 \%$ \\
Somewhat important & $35.7 \%$ \\
Not very important & $16.0 \%$ \\
Not at all important & $4.7 \%$ \\
\hline
\end{tabular}




\section{EXTENDED SIMULATION RESUlTS}

\subsection{Additional simulation scenarios}

The following figures show all results presented in the main text as well as additional scenarios.

Figure 1: 95\% null intervals versus mean rejections in observed datasets $(\times)$. Panels: Null and alternative data-generating mechanisms of original samples. Points and error bars: Mean $\widehat{\theta}^{(j)}$ and mean limits of null intervals with tests at $\alpha=0.01$ (yellow) or at $\alpha=0.05$ (red).

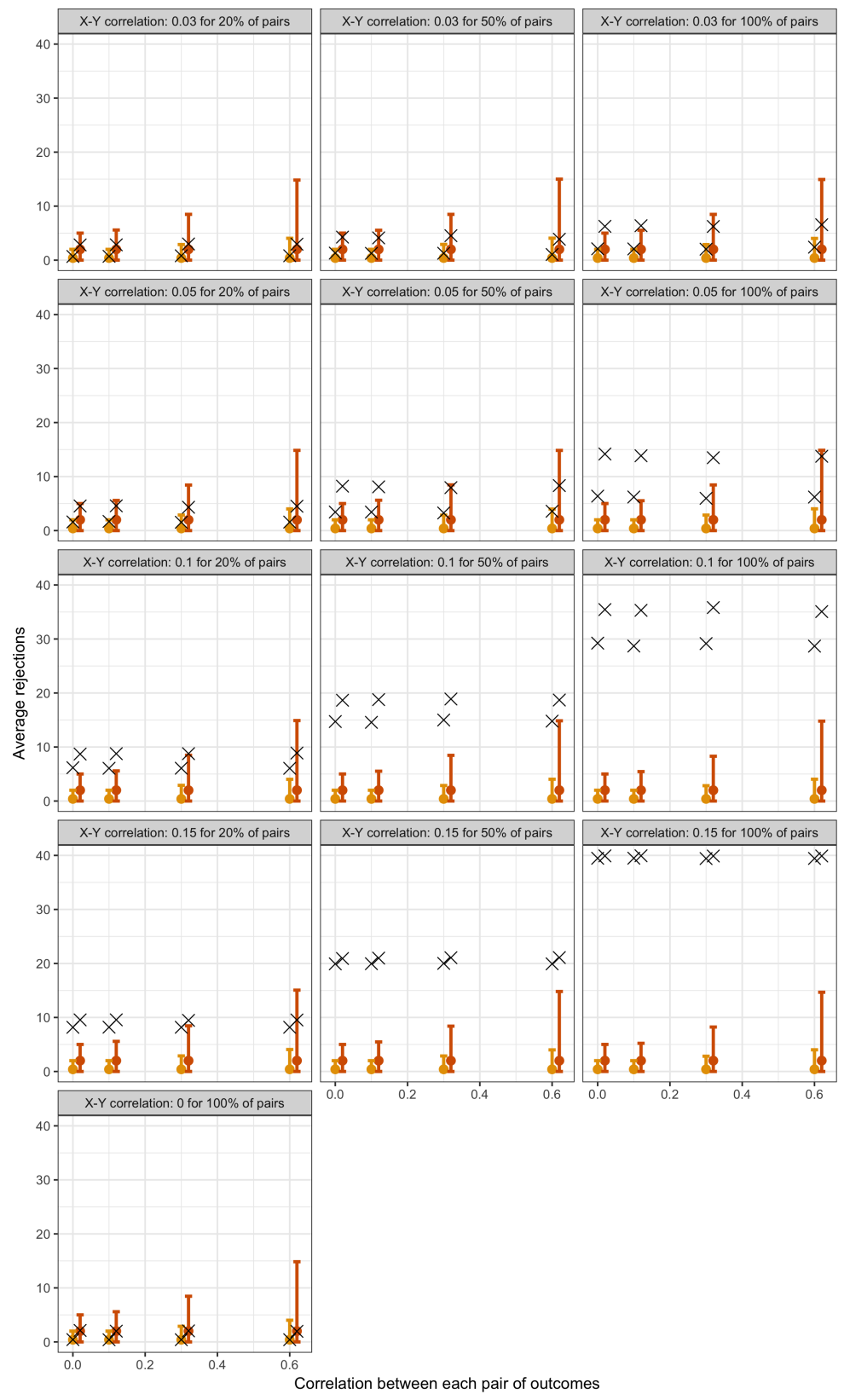


Figure 2: Power of global tests based on existing FWER-control procedures and on the number of rejections. $B=$ Bonferroni, $H=H o l m, M P=\min P, W S=$ Wstep, $R=$ Romano, $G 1=$ number of rejections at $\alpha=0.01, G 5=$ number of rejections at $\alpha=0.05$.

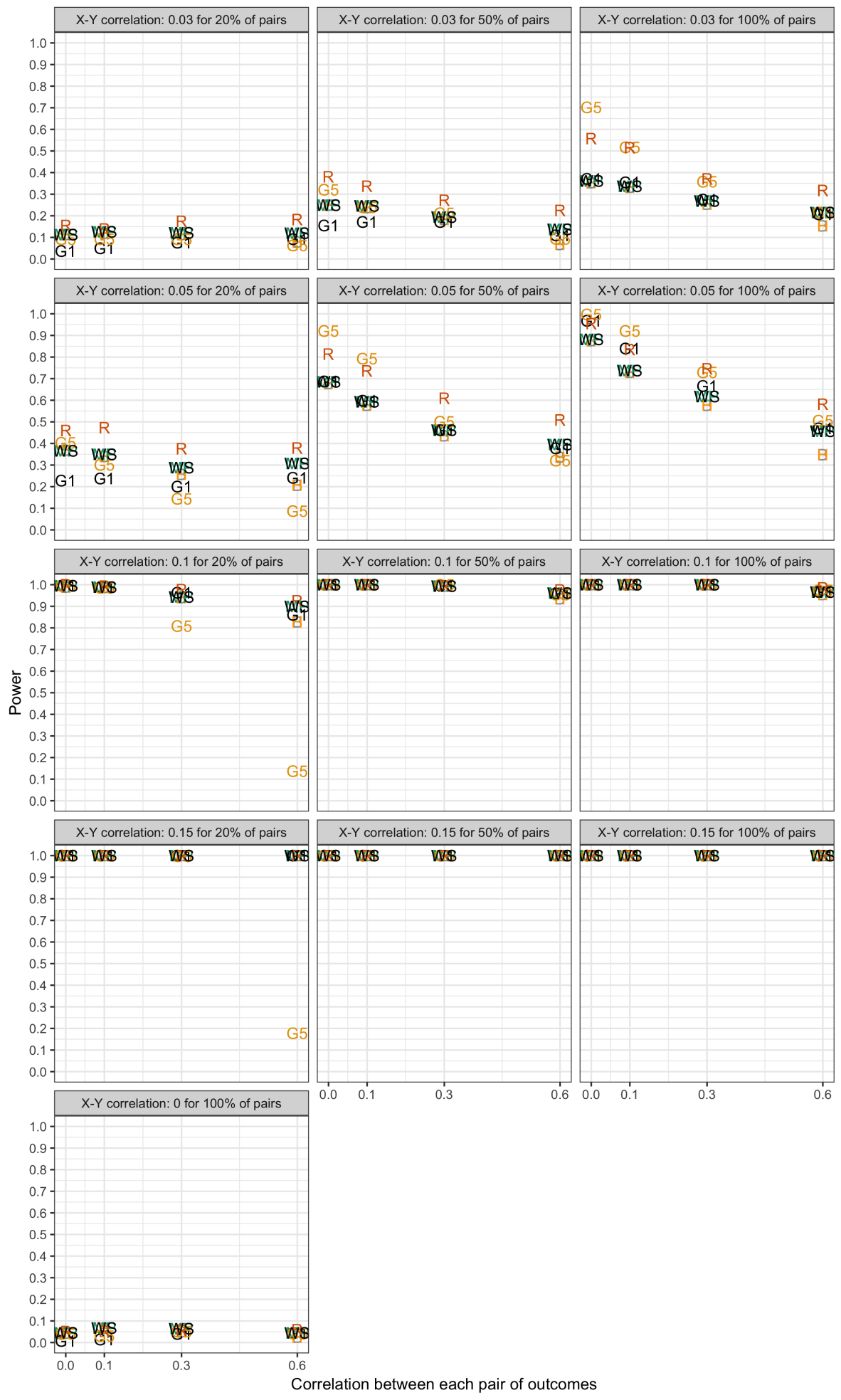


Figure 3: Number of rejected null hypotheses at familywise-controlled $\alpha_{W}=0.05$ based on existing $F W E R$-control procedures and on the excess hits. $B=$ Bonferroni, $H=H o l m, M P=\operatorname{minP}$, $W S=$ Wstep,$R=$ Romano, $G 1=$ number of rejections at $\alpha=0.01$, G5=number of rejections at $\alpha=0.05$. Red dashed line: Actual number of false null hypotheses $(q \times W)$.
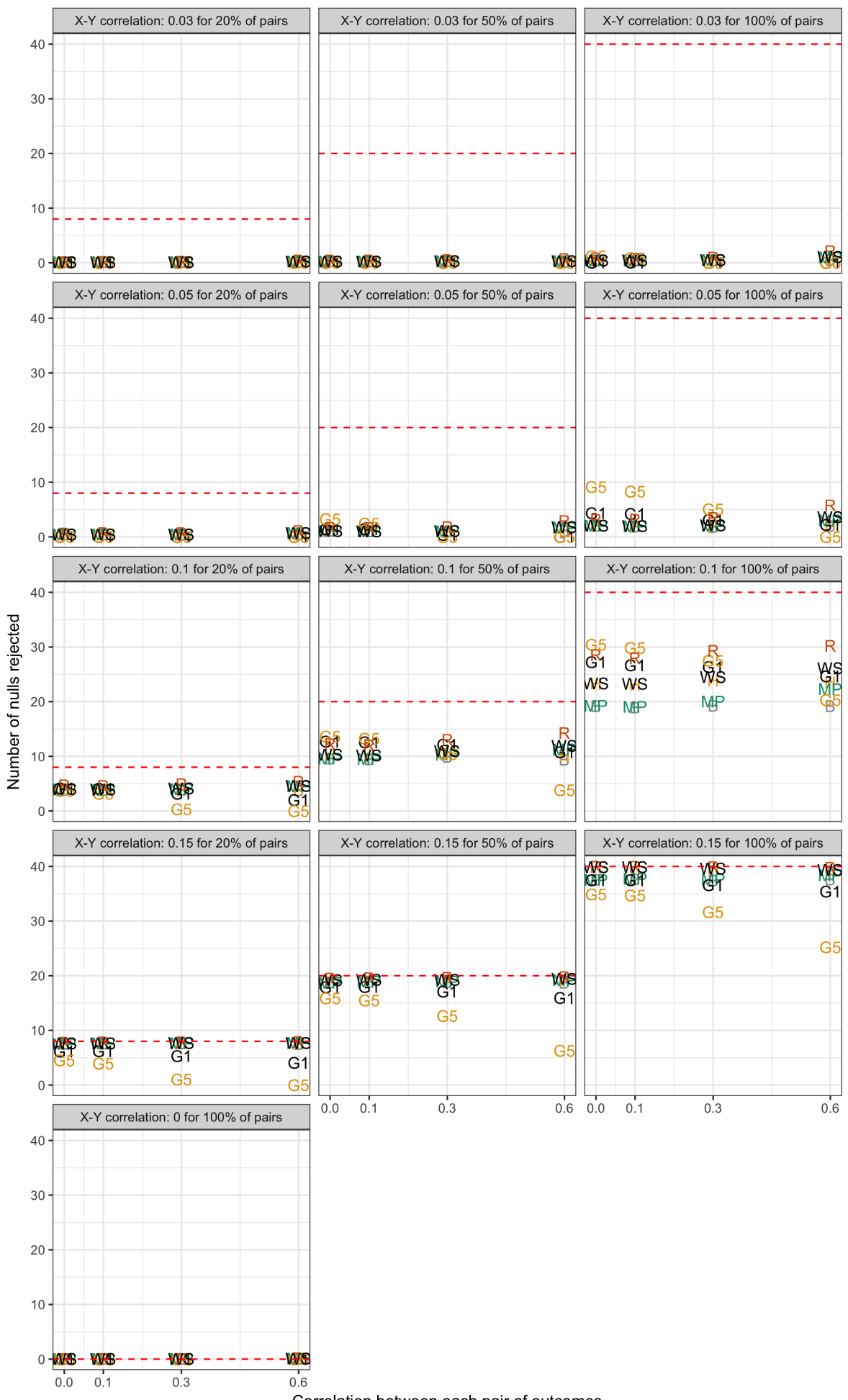

Correlation between each pair of outcomes 


\subsection{Comparison of $p$-values adjusted by existing methods}

We performed a rudimentary visual comparison of $p$-value adjustments produced by one naïve method (Holm) and one resampling-based method (Wstep). We generated a single dataset as in the simulation study with 1 covariate, 100 outcomes, $N=1,000, \rho_{X Y}=0.08$ for all outcomes, and $\rho_{Y Y}=0.25$. We chose these parameters to yield a large number of adjusted $p$-values $<0.05$ for illustrative purposes. Figure 4 plots the $100 p$-values adjusted using the Holm and Wstep methods (obtained by resampling as in the applied example with $B=500$ resamples) and suggests that in this simple simulation, the methods differ little in their adjustments to $p$-values near $\alpha=0.05$; rather, the differences appear to emerge primarily for $p>>0.05$. We obtained qualitatively similar results when comparing other pairs of existing methods (not shown).

Figure 4: $p$-values in a single simulated dataset adjusted by the Holm method versus the Wstep method. Red dashed lines: $\alpha=0.05$ threshold.

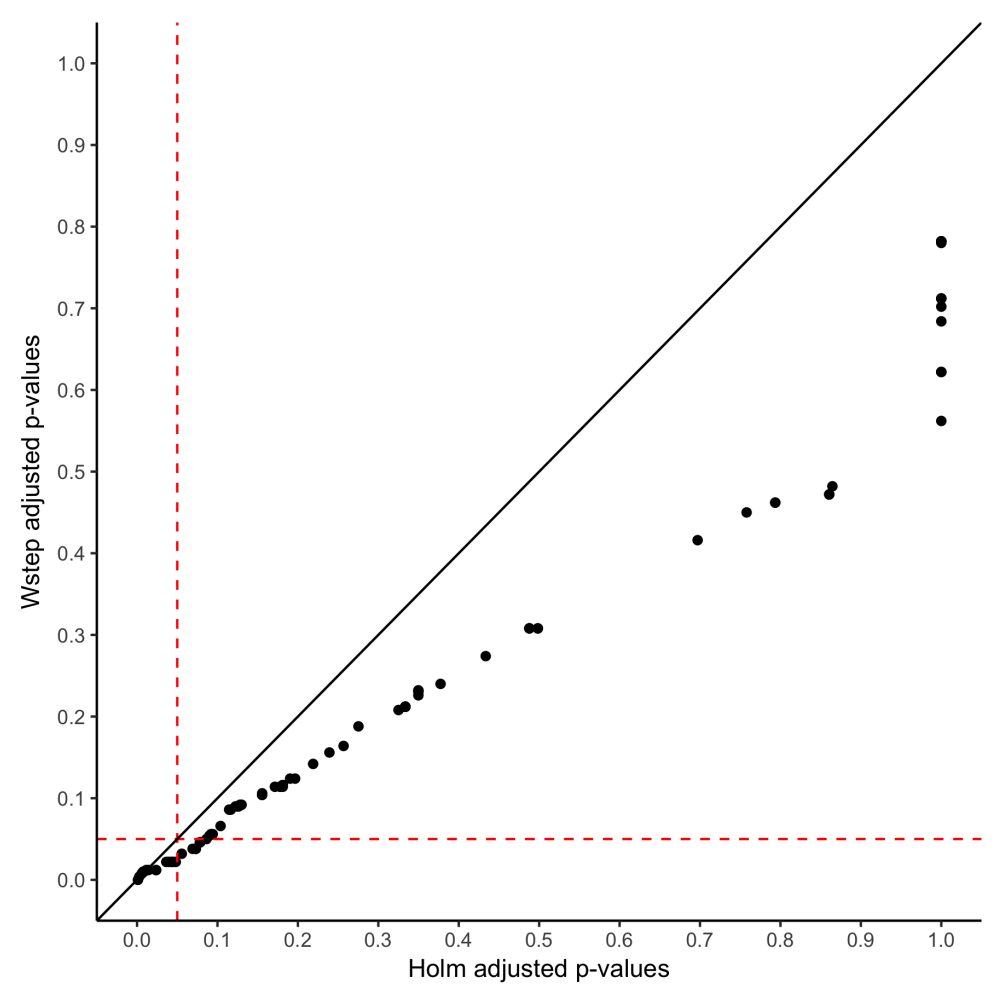




\section{INTRODUCTION TO THE PACKAGE NREJECTIONS}

Here we briefly describe the $\mathrm{R}$ package NRejections; note that additional functions, details, and additional examples are available in the standard $\mathrm{R}$ documentation. For OLS models as described in Section 4.2 of the main text, the null interval, excess hits, and global test can be conducted by calling a single wrapper function, corr_tests. This function first fits the $W$ models in the original dataset, adjusting for any user-specified covariates. Then, resamples are generated via Algorithm 4.1 (main text) and used to estimate and return our proposed metrics, along with estimates and inference from the original sample. Optionally, the global test can additionally be conducted using any combination of methods in Table 1 (main text). Below is a minimal example.

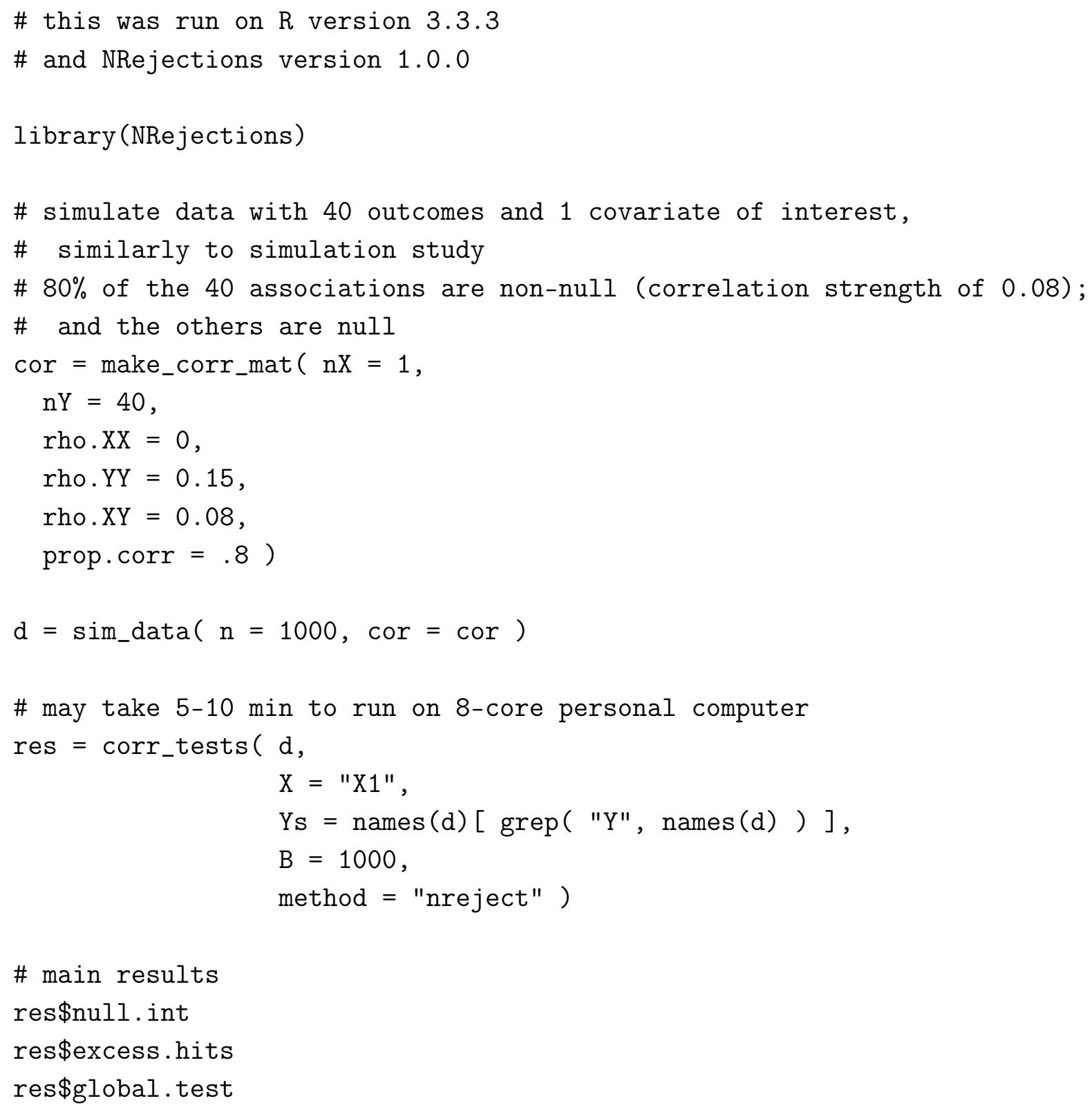


As described in the Discussion, Algorithm 4.1 is more broadly applicable to multipletesting procedures outside the scope of this paper. For these general applications, the user could first obtain residuals and point estimates from the original dataset using the function dataset_result and pass these to resid_resample, which returns matrices of $p$-values and test statistics from the resamples. See ?resid_resample for examples. 


\section{REFERENCES}

Bickel, P. J., \& Freedman, D. A. (1981). Some asymptotic theory for the bootstrap. The Annals of Statistics, 1196-1217.

DasGupta, A. (2008). Asymptotic theory of statistics and probability. Springer, New York.

Freedman, D. A. (1981). Bootstrapping regression models. The Annals of Statistics, 1218-1228. 